\title{
Estructura y composición de los ensamblajes estacionales de coleópteros (Insecta: Coleoptera) del bosque mesófilo de montaña en Tlanchinol, Hidalgo, México, recolectados con trampas de intercepción de vuelo
}

\section{Structure and composition of seasonal coleopterous assemblages (Insecta: Coleoptera) in the cloud forest of Tlanchinol, Hidalgo, Mexico collected with flight interception traps}

\author{
Ma. del Carmen Pedraza ${ }^{1}$, Juan Márquez ${ }^{2 *}$ y José. A. Gómez-Anaya ${ }^{3}$ \\ ${ }^{1}$ Calle 23 de Marzo 18, Col. Independencia, Tlanchinol 43150, Hidalgo, México. \\ ${ }^{2}$ Laboratorio de Sistemática Animal, Centro de Investigaciones Biológicas, Universidad Autónoma del Estado de Hidalgo. Km. 4.5 carretera \\ Pachuca-Tulancingo s/n, Ciudad Universitaria, 42184 Pachuca Hidalgo, México. \\ ${ }^{3}$ Instituto de Ecología, A.C. Km. 2.5 carretera antigua a Coatepec 351, Congregación El Haya, 91070, Xalapa Veracruz, México. \\ *Correspondencia: jmarquez@uaeh.edu.mx
}

\begin{abstract}
Resumen. Se describen y comparan los ensamblajes de coleópteros de las temporadas de lluvias y sequía del bosque mesófilo de montaña de Tlanchinol, Hidalgo, que se obtuvieron en 8 recolectas mensuales usando trampas de intercepción de vuelo. Para la descripción de los ensamblajes se tomó en cuenta su riqueza, composición, diversidad, equidad y dominancia y se compararon con base en los perfiles de diversidad de Renyi. El recambio de especies se evaluó usando el índice de similitud de Bray-Curtis. Se estimó la riqueza teórica del ensamblaje total y por temporada, se exploró la relación entre los taxa y los meses de recolecta, y las especies se ubicaron en grupos funcionales. Los 3308 ejemplares recolectados pertenecen a 50 familias y 352 especies, siendo Staphylinidae, Curculionidae y Nitidulidae las familias con el mayor número de especies. La riqueza, abundancia y diversidad de la época de sequía fueron significativamente mayores que las de lluvias. La mayoría de las especies poseen abundancias inferiores a 1\% conformando las especies raras. Teóricamente aún faltan taxa por recolectarse en Tlanchinol. La mayoría de las especies se ubicaron en la categoría de los depredadores (43\%) y fitófagos (20\%). Los resultados resaltan la gran biodiversidad de estos bosques y su urgente necesidad de protección.
\end{abstract}

Palabras clave: Tlanchinol, Hidalgo, bosque mesófilo de montaña, Coleoptera, diversidad, conservación.

\begin{abstract}
We described and compared Coleoptera assemblages in rainy and dry seasons in the cloud forest in Tlanchinol, Hidalgo, Mexico, based on 8 monthly samples using flight interception traps. Assemblages were described using richness and composition as well as some diversity measurements; we then used Renyi's diversity profiles to compare them. A BrayCurtis similarity index was used to evaluate species turnover rate between seasons. The theoretical richness by parametric methods was estimated, the relationship between species and the collecting months were analyzed with a correspondence analysis, and species were placed into functional groups. The 3308 specimens collected belong to 50 families and 352 species, with Staphylinidae, Curculionidae and Nitidulidae having the highest number of species. Richness, abundance and diversity were significantly higher in the dry season. Most species possess abundances lower than $1 \%$ representing the rare species. According to the theoretical estimation of richness there are many species to add to the list. Predators $(43 \%)$ and phytophagous (20\%) comprised the highest number of species. The great diversity of surveyed coleopterous represents an important factor in the Tlanchinol cloud forest protection.
\end{abstract}

Key words: Tlanchinol, Hidalgo, cloud forest, Coleoptera, diversity, conservation.

\section{Introducción}

La diversidad biológica es actualmente un tema central de la teoría ecológica y ha sido objeto de amplios debates (Magurran, 1988); para conservarla, se requiere del

Recibido: 15 mayo 2009; aceptado: 30 octubre 2009 conocimiento de sus patrones y de su magnitud (Baselga y Novoa, 2008), donde el proceso de inventariar ensamblajes desconocidos y describir nuevas especies representa el primer paso en la ciencia taxonómica y es la base para cualquier investigación relacionada con la biodiversidad.

En grupos megadiversos, como Coleoptera, los inventarios distan mucho de ser completos, lo que en 
parte se debe a la gran cantidad de especies que aún no están descritas (May, 1988; Erwin, 1991; Odegaard, 2000) y al relativamente poco valor que se le da a ese tipo de trabajo en las revistas internacionales de biodiversidad, propiciando su abandono por los investigadores (Wheeler, 2004; Wheeler et al., 2004; de Carvalho et al., 2005).

Con fines de conservación, manejo y monitoreo ambiental se han desarrollado métodos para medir la biodiversidad, como un indicador del estado de los ecosistemas (Spellerberg, 1991). El número de especies es la medida que más frecuentemente se utiliza (Mayr, 1992; Moreno y Halffter, 2000); sin embargo, la biodiversidad no depende sólo de la riqueza de especies, sino también de la dominancia relativa de cada una de ellas. En general, las especies se distribuyen según jerarquías de abundancia, desde muy abundantes hasta muy raras. Cuanto mayor es el grado de dominancia de ciertas especies y de rareza de las demás, menor es la biodiversidad de la comunidad. La conservación de la biodiversidad es principalmente un problema vinculado al comportamiento ecológico de las especies raras. De esta manera, medir la abundancia relativa de cada especie permite identificar las que por su escasa representatividad en la comunidad son más sensibles a las perturbaciones ambientales (Moreno, 2001). Ante el actual empobrecimiento de la diversidad biológica es indispensable trabajar en líneas de investigación que se ocupen de la recolecta de organismos y con ello formar inventarios biológicos completos que representen mejor la biodiversidad y la distribución geográfica de cada grupo biológico, incluyendo en especial los menos conocidos (Sánchez-Cordero et al., 2001).

El bosque mesófilo de montaña (BMM) es un tipo de bosque derivado de los cambios climáticos del pleistoceno que posee una elevada diversidad biológica. Desafortunadamente en México sólo existen parches, ya que principalmente por la tala intensiva y la agricultura se ha visto reducido y fragmentado hasta llegar a representar menos del 1\% del territorio nacional (Luna et al., 2004). A pesar de ello, en ese pequeño porcentaje está albergado cerca del $12 \%$ de la flora total del país. Estos bosques son santuarios de especies amenazadas y contienen un alto porcentaje de endemismos, pero es uno de los ecosistemas más seriamente amenazados y en peligro de extinción (Challenger, 1998; Luna et al., 2001). Hidalgo es el tercer estado de la República Mexicana con mayor superficie ocupada por BMM (Luna et al., 2004), contiene el 34\% de la riqueza florística considerada para este tipo de vegetación en el país, y dentro del estado de Hidalgo, los bosques de Tlanchinol son los que se estiman como mejor conservados (Luna et al., 1994).

No existen estudios generales sobre coleópteros del municipio de Tlanchinol, aunque sí varios que analizan los coleópteros Scarabaeoidea (Morón y Terrón, 1984, 1986; Delgado y Márquez, 2006) y Staphylinidae (Asiain y Márquez, 2003; Márquez, 2003, 2006; FierrosLópez, 2005). Los objetivos de este estudio son describir y comparar los ensamblajes de coleópteros de las temporadas de lluvias y sequía recolectados mediante el uso de trampas de intercepción de vuelo (TIV), así como estimar la riqueza potencial de este grupo en el BMM de Tlanchinol, Hidalgo.

Se utilizaron trampas de intercepción de vuelo, también conocidas como trampas de ventana, debido a que son un sencillo y productivo método de muestreo con el que se recolecta una gran diversidad y número de insectos (Peck y Davies, 1980; Márquez, 2005). La mayoría de los trabajos publicados se han enfocado a la recolección de todo tipo de artrópodos, pero ha sido notable su eficiencia para la recolección de coleópteros (Juillet, 1963; Hosking, 1979; Basset, 1988; Basset y Arthington, 1992; Hill y Cermak 1997; McWilliam y Death, 1998; Leksono et al., 2005).

\section{Materiales y métodos}

Zona de estudio. El municipio de Tlanchinol se ubica en la parte norte del estado de Hidalgo, entre las coordenadas geográficas $19^{\circ} 59^{\prime} 21^{\prime \prime} \mathrm{N}$ y $98^{\circ} 40^{\prime} 43^{\prime}$ 'O. Se encuentra a una altitud promedio de $1590 \mathrm{~m}$ y ocupa $380 \mathrm{~km}^{2}$ (SEDESOL, 2002). La localidad de estudio se denomina La Cabaña, está ubicada a $2.5 \mathrm{~km}$ al NE de la cabecera municipal, entre $\operatorname{los} 21^{\circ} 01.357^{\prime} \mathrm{N}$ y $98^{\circ} 38.556^{\prime} \mathrm{O}$, a una altitud de $1485 \mathrm{~m}$ (Fig. 1). Predomina el clima semicálidosubhúmedo, con temperatura media anual de $18.9^{\circ} \mathrm{C}$, precipitación pluvial anual de $2601 \mathrm{~mm}$ (Luna et al., 1994; SEDESOL, 2002) y lluvias en los meses de mayo a septiembre. El municipio se encuentra ubicado sobre una superficie abrupta, lodosa, húmeda, con muchas barrancas, mesetas y valles, dentro de la región del río Pánuco y la cuenca del río Moctezuma. Por el municipio pasan los ríos Tehuetlán, Santa María, Xalpan, Amajac y Quetzaltongo (SEDESOL, 2002).

En el área de estudio se presenta el bosque mesófilo de montaña, con árboles que alcanzan alturas entre 30 y 35 m. Rzedowski (1991, en Luna et al., 1994) estimó que Tlanchinol representaba el $11.5 \%$ de la riqueza florística estimada para este tipo de vegetación en México.

Muestreo. Se instalaron 5 trampas de intercepción de vuelo (TIV), dispersas en una zona del bosque con un grado de deforestación bajo, separadas $50 \mathrm{~m}$ entre sí. Este tipo de trampas constan de una cortina que se coloca en posición vertical respecto a la vegetación. Las trampas se elaboraron con hule transparente con la finalidad de que no fueran visibles a los organismos que vuelan por 


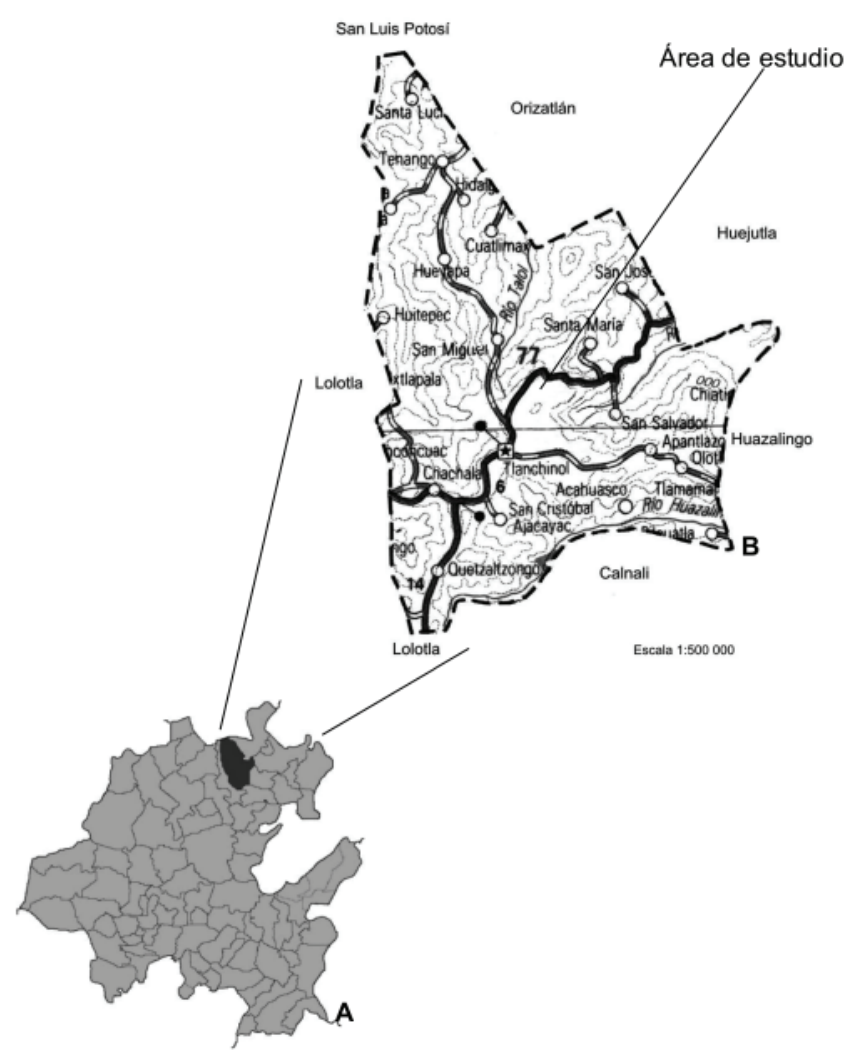

Figura 1. Ubicación geográfica del área de estudio. A. Mapa estatal (tomado de SEDESOL, 2002); B. Mapa municipal (modificado de SCT 1995).

el lugar, propiciando su impacto contra el hule y su caída a recipientes recolectores que contenían una solución de agua y jabón para impedir que retomaran el vuelo (Márquez, 2005).

Los muestreos se efectuaron durante 2 periodos: época de lluvias (junio-septiembre de 2005) y época de sequía (febrero-mayo de 2006). Las trampas se colocaron la tercera semana de cada mes de recolección y permanecieron instaladas 8 días, pasados los cuales se vació el contenido de cada recipiente a un frasco con alcohol etílico al $70 \%$, pasando antes por un colador de tela fina, para que los organismos más pequeños no cayeran al suelo. Se obtuvieron 40 muestras en total.

Trabajo de laboratorio. Los coleópteros fueron separados del resto de los insectos y se colocaron en viales con alcohol al 70\%, con su etiqueta respectiva. Posteriormente, se separaron en morfoespecies y se cuantificaron. Se montaron como mínimo 2 organismos de cada morfoespecie (exceptuando las representadas por un solo individuo), para facilitar su observación al microscopio.

La identificación de los organismos se realizó al nivel taxonómico más fino posible, con ayuda de la bibliografía (Arnett y Thomas, 2001; Arnett et al., 2002; Morón et al., 1997; Morón, 2003; Navarrete-Heredia et al., 2002 y White, 1983). Para el nivel de familia y subfamilia se utilizó el criterio de clasificación de Lawrence y Newton (1995). El trabajo de identificación se complementó con la ayuda de diferentes especialistas.

De los coleópteros recolectados, 11 organismos de las 3 morfoespecies de Phengodidae y 2 de la única morfoespecie de Telegeusidae quedaron depositados en la Colección Nacional de Insectos del Instituto de Biología, Universidad Nacional Autónoma de México (CNIN). El resto se depositó en la colección de Coleoptera del Centro de Investigaciones Biológicas, Universidad Autónoma del Estado de Hidalgo (CC-UAEH).

Análisis de datos. Los ensamblajes de las épocas de lluvias y sequía fueron descritos en términos de su riqueza (número de morfoespecies) y composición; adicionalmente, se calcularon los siguientes índices: diversidad de Shannon (H'), diversidad de Simpson (D) como medida de dominancia, riqueza de Margalef $\left(R_{M}\right)$ y equitatividad de Pielou (Moreno, 2001). Para comparar la diversidad de los ensamblajes se utilizaron los perfiles de diversidad de Renyi (Tóthmérész, 1998) generados en una hoja de cálculo de Excel. El cálculo se basó en la frecuencia relativa de presencia de las morfoespecies. Como caso especial, este índice incluye el logaritmo del número de especies $(\alpha=0)$, el índice de diversidad de Shannon $(\alpha=1)$, el índice de diversidad de Simpson $(\alpha=2)$ y el índice de diversidad de Berger-Parker cuando $\alpha$ toma valores muy grandes (Tóthmérész, 1995).

Con el fin de estimar la riqueza potencial del área de estudio y evaluar la eficiencia del muestreo se utilizaron las funciones de acumulación de especies de Clench y dependencia lineal (Soberón y Llorente, 1993), las cuales fueron ajustadas en el programa Statistica v.7 (Statsoft, 1998) mediante el procedimiento de regresión no lineal definida por el usuario. Se utilizó el método de ajuste Simplex \& Quasi-Newton que, de acuerdo con JiménezValverde y Hortal (2003), es uno de los más robustos. Lo anterior se realizó a partir de la columna de número promedio de especies acumuladas, generada con el programa Estimates v 7.5. (Colwell, 2005).

Como criterio para evaluar la completitud del listado final se observó la magnitud de la pendiente de las curvas para el esfuerzo total de muestreo $(n=40)$; dichas pendientes se calcularon mediante las primeras derivadas de las 2 funciones (Hortal y Lobo, 2005). Ambas han sido usadas para estimar la riqueza teórica de ensamblajes de coleópteros (Escobar, 2000; Morón-Ríos y Morón, 2001; Lobo y Piera, 2001; Reyes-Novelo y Morón, 2005; González y Basélga, 2007; Reyes-Novelo et al., 2007; 
Baselga y Novoa, 2008).

Para probar el efecto de la temporada (lluvias y sequía) sobre la abundancia de coleópteros, se utilizó un ANOVA no paramétrico o prueba de Kruskal Wallis después de encontrar que los datos no se distribuían normalmente (prueba de Shapiro-Wilk) y que las varianzas no eran homogéneas (Zar, 1996). De la misma forma, se aplicó esta prueba para probar el efecto del factor mes sobre la abundancia de coleópteros. En este caso, y de haber efecto significativo, se procedió a utilizar la prueba $U$ de Mann-Whitney con el fin de discernir donde radicaban las diferencias.

La similitud faunística entre los meses de recolección (Cuadro 1) fue evaluada mediante el índice de similitud de Bray-Curtis (1-W), donde $\mathrm{W}=$ disimilitud de BrayCurtis y se calcula como $\mathrm{W}=2 p N / \mathrm{a} N+\mathrm{b} N, p N=$ sumatoria de la abundancia más baja de cada una de las especies compartidas, $a N=$ número total de individuos en el sitio A y $b N=$ número de individuos en el sitio B (Moreno, 2001). Para este fin se utilizó el programa gratuito PAST. De igual forma, se aplicó un análisis de correspondencias simple (AC) a una tabla de contingencia que contenía las frecuencias de presencia de las morfoespecies para cada mes de recolección. El resultado fue un diagrama cartesiano basado en la asociación entre las variables analizadas (Manly, 1998) que permitió visualizar el grado de asociación de las morfoespecies con los meses de recoleccción.

Finalmente, las morfoespecies se ubicaron en grupos funcionales con base en sus hábitos alimenticios (White, 1983; Morón et al., 1997; Arnet y Thomas, 2001; Arnet et al., 2002; Navarrete-Heredia et al., 2002; Morón, 2003): fitófagos (FI), los organismos que se alimentan de cualquier parte de las plantas; micófagos (MI), los que se alimentan de cualquier parte de los hongos; depredadores (DE), los que cazan a sus presas para alimentarse de ellas; saprófagos (SA), los que se alimentan de cualquier tipo de materia orgánica en descomposición; necrófagos (NE),

Cuadro 1. Similitud faunística pareada de Bray-Curtis entre los ensamblajes mensuales de coleópteros de Tlanchinol, Hidalgo

\begin{tabular}{lllllllll}
\hline & jun & jul & ags & sep & feb & mar & abr & may \\
\hline jun & 1.00 & & & & & & & \\
jul & 0.50 & 1.00 & & & & & & \\
ags & 0.40 & 0.45 & 1.00 & & & & & \\
sep & 0.31 & 0.37 & 0.40 & 1.00 & & & & \\
feb & 0.16 & 0.18 & 0.31 & 0.25 & 1.00 & & & \\
mar & 0.13 & 0.21 & 0.20 & 0.22 & 0.31 & 1.00 & & \\
abr & 0.18 & 0.24 & 0.31 & 0.21 & 0.32 & 0.33 & 1.00 & \\
may & 0.21 & 0.31 & 0.37 & 0.29 & 0.37 & 0.24 & 0.42 & 1.00 \\
\hline
\end{tabular}

los que se alimentan de animales muertos; coprófagos (CO), los que se alimentan de excremento de vertebrados; saprófagos/micófagos (SA/MI), los que se alimentan de materia orgánica en descomposición y/o de cualquier parte de los hongos; depredadores/micófagos (DE/MI), los que se alimentan cazando a sus presas o pueden alimentarse de hongos, sin que se considere que pueden alimentarse de los 2 recursos, pero que en la literatura consultada se incluyen ambas posibilidades para un grupo determinado; omnívoros (OM), los que pueden consumir una variedad amplia de alimentos. Se consideraron "desconocidos" o indeterminados (IN) los organismos de cuyos hábitos alimenticios no se encontró información alguna. La mayoría de las morfoespecies se ubicaron en alguno de los anteriores grupos.

\section{Resultados}

Se obtuvieron 3308 ejemplares pertenecientes a 50 familias. Se determinaron 20 especies, 98 géneros, 6 tribus, 94 subfamilias y 134 familias, totalizando 352 morfoespecies (Cuadro 2). De manera general, la morfoespecie dominante del ensamblaje fue Aleocharinae sp. 8 (Staphylinidae) con el $12.18 \%$ de la abundancia total, mientras que Thalycra sp. 1 (Nitidulidae) y Scydmaenidae sp. 1 (Scydmaenidae) fueron especies codominantes. El número de especies con abundancias relativas inferiores a $1 \%$ fue elevado $(93.18 \%)$.

La mayoría de las familias estuvieron representadas por pocas morfoespecies. Las familias con mayor número de morfoespecies fueron Staphylinidae con 123, Curculionidae con 30 y Nitidulidae con 20 (Fig. 2). Entre junio y septiembre se registraron menos morfoespecies, $y$ a partir de febrero hubo un incremento notable (Fig. 3).

Los índices de diversidad indicaron una mayor riqueza de taxa durante la época de sequía $\left(\mathrm{R}_{\mathrm{M}}=39.94\right)$ que en lluvias $\left(\mathrm{R}_{\mathrm{M}}=22.81\right)$. Asimismo, durante las secas la abundancia fue poco más del doble (2 290 individuos) que durante las lluvias (1 018 individuos). La equidad mostró un reparto similar de la abundancia entre las morfoespecies (sequía, $\mathrm{J}=0.79$; lluvias, $\mathrm{J}=0.80$ ); por esta razón, la diversidad de Shannon fue apenas mayor durante las secas $\left(\mathrm{H}^{\prime}=4.57\right)$ que en lluvias $\left(\mathrm{H}^{\prime}=4.01\right)$ (Fig. 4).

Los perfiles de diversidad de Renyi (Fig. 5) mostraron que la diversidad de la época de sequía (2006) es superior a la de lluvias (2005), debido a que dichos perfiles no se cruzan en ningún punto en todo el rango de $\alpha$. Los ensamblajes son principalmente diferentes en el nivel de su estructura básica, ésto es, en el número de especies $(\alpha=0)$. Durante la época de lluvias, la estructura de la dominancia numérica mostró a Thalycra sp. 1 (Nitidulidae) como la 
Cuadro 2. Ensamblajes estacionales de coleópteros capturados con trampas de intercepción de vuelo en Tlanchinol, Hidalgo, México

\begin{tabular}{|c|c|c|c|c|c|c|c|c|c|c|c|}
\hline $\begin{array}{l}\text { Clave } \\
\text {-taxon }\end{array}$ & & $\begin{array}{l}\text { Hábitos } \\
\text { aliment. }\end{array}$ & & Lluvias & & & Secas & & & Total & \\
\hline & & & $A b d$ & $\%$ & Frec & $A b d$ & $\%$ & Frec & $A b d$ & $\%$ & Frec \\
\hline & Número de taxa & & 159 & & & 310 & & & 352 & & \\
\hline & Abundancia & & 1018 & & & 2290 & & & 3308 & & \\
\hline & ANOBIIDAE & & & & & & & & & & \\
\hline 1 & Anobiidae sp. 1 & FI & 1 & 0.10 & 1 & - & - & - & 1 & 0.03 & 1 \\
\hline 2 & Anobiidae sp. 2 & FI & - & - & - & 2 & 0.09 & 2 & 2 & 0.06 & 2 \\
\hline 3 & Anobiidae sp. 3 & FI & - & - & - & 1 & 0.04 & 1 & 1 & 0.03 & 1 \\
\hline 4 & $\begin{array}{l}\text { Anobiidae sp. } 4 \\
\text { ANTHICIDAE }\end{array}$ & FI & - & - & - & 1 & 0.04 & 1 & 1 & 0.03 & 1 \\
\hline 5 & $\begin{array}{l}\text { Anthicidae sp. } \\
\text { BRENTIDAE }\end{array}$ & $\mathrm{OM}$ & - & - & - & 1 & 0.04 & 1 & 1 & 0.03 & 1 \\
\hline 6 & $\begin{array}{l}\text { Apioninae sp. } \\
\text { BIPHYLLIDAE }\end{array}$ & FI & 1 & 0.10 & 1 & 1 & 0.04 & 1 & $\begin{array}{l}2 \\
-\end{array}$ & $\begin{array}{l}0.06 \\
-\end{array}$ & $\begin{array}{l}2 \\
-\end{array}$ \\
\hline 7 & $\begin{array}{l}\text { Diplocoelus sp. } \\
\text { CANTHARIDAE }\end{array}$ & MI & - & - & - & 3 & 0.13 & 2 & 3 & 0.09 & 2 \\
\hline 8 & Malthinus sp. & $\mathrm{OM}$ & & & & 1 & 0.04 & 1 & 1 & 0.03 & 1 \\
\hline 9 & Discodon sp. 1 & $\mathrm{OM}$ & 1 & 0.10 & 1 & 3 & 0.13 & 3 & 4 & 0.12 & 4 \\
\hline 10 & Discodon sp. 2 & $\mathrm{OM}$ & 1 & 0.10 & 1 & & & & 1 & 0.03 & 1 \\
\hline 11 & $\begin{array}{l}\text { Silis sp. } \\
\text { CARABIDAE }\end{array}$ & $\mathrm{OM}$ & 2 & 0.20 & 2 & 1 & 0.04 & 1 & 3 & 0.09 & 3 \\
\hline 12 & Carabidae sp. 1 & $\mathrm{DE}$ & 4 & 0.39 & 2 & - & - & - & 4 & 0.12 & 2 \\
\hline 13 & Carabidae sp. 2 & $\mathrm{DE}$ & 4 & 0.39 & 3 & 7 & 0.31 & 6 & 11 & 0.33 & 9 \\
\hline 14 & Carabidae sp. 3 & $\mathrm{DE}$ & 8 & 0.79 & 4 & 37 & 1.62 & 11 & 45 & 1.36 & 15 \\
\hline 15 & Carabidae sp. 4 & $\mathrm{DE}$ & 4 & 0.39 & 3 & 8 & 0.35 & 5 & 12 & 0.36 & 8 \\
\hline 16 & Carabidae sp. 5 & $\mathrm{DE}$ & 14 & 1.38 & 7 & 18 & 0.79 & 6 & 32 & 0.97 & 13 \\
\hline 17 & Carabidae sp. 6 & $\mathrm{DE}$ & 3 & 0.29 & 2 & - & - & - & 3 & 0.09 & 2 \\
\hline 18 & Carabidae sp. 7 & $\mathrm{DE}$ & - & - & - & 1 & 0.04 & 1 & 1 & 0.03 & 1 \\
\hline 19 & Carabidae sp. 8 & $\mathrm{DE}$ & - & - & - & 2 & 0.09 & 2 & 2 & 0.06 & 2 \\
\hline 20 & Carabidae sp. 9 & $\mathrm{DE}$ & - & - & - & 1 & 0.04 & 1 & 1 & 0.03 & 1 \\
\hline 21 & $\begin{array}{l}\text { Carabidae sp. } 10 \\
\text { CERAMBYCIDAE }\end{array}$ & $\mathrm{DE}$ & - & - & - & 1 & 0.04 & 1 & 1 & 0.03 & 1 \\
\hline 22 & Cerambicidae sp. 1 & FI & - & - & - & 1 & 0.04 & 1 & 1 & 0.03 & 1 \\
\hline 23 & $\begin{array}{l}\text { Cerambicidae sp. } 2 \\
\text { CERATHOCANTIDAE }\end{array}$ & FI & - & - & - & 1 & 0.04 & 1 & 1 & 0.03 & 1 \\
\hline 24 & Cerathocantidae sp. 1 & SA/MI & 6 & 0.59 & 3 & 5 & 0.22 & 2 & 11 & 0.33 & 5 \\
\hline 25 & $\begin{array}{l}\text { Cerathocantidae sp. } 2 \\
\text { CERYLONIDAE }\end{array}$ & SA/MI & - & - & - & 1 & 0.04 & 1 & 1 & 0.03 & 1 \\
\hline 26 & $\begin{array}{l}\text { Cerylonidae sp. } \\
\text { CHRYSOMELIDAE }\end{array}$ & MI & - & - & - & 1 & 0.04 & 1 & 1 & 0.03 & 1 \\
\hline 27 & Chrysomelinae sp. 1 & FI & 1 & 0.10 & 1 & - & - & - & 1 & 0.03 & 1 \\
\hline 28 & Chrysomelinae sp. 2 & FI & - & - & - & 1 & 0.04 & 1 & 1 & 0.03 & 1 \\
\hline 29 & Chrysomelinae sp. 3 & FI & 2 & 0.20 & 2 & 1 & 0.04 & 1 & 3 & 0.09 & 3 \\
\hline 30 & Chrysomelinae sp.4 & FI & - & - & - & 5 & 0.22 & 4 & 5 & 0.15 & 4 \\
\hline 31 & Chrysomelinae sp. 5 & FI & - & - & - & 6 & 0.26 & 5 & 6 & 0.18 & 5 \\
\hline 32 & Eumolpinae sp. 1 & FI & - & - & - & 2 & 0.09 & 2 & 2 & 0.06 & 2 \\
\hline 33 & Eumolpinae sp. 2 & FI & - & - & - & 3 & 0.13 & 3 & 3 & 0.09 & 3 \\
\hline 34 & Eumolpinae sp. 3 & FI & - & - & - & 1 & 0.04 & 1 & 1 & 0.03 & 1 \\
\hline 35 & Chrysomelidae sp. 1 & FI & 2 & 0.20 & 2 & 1 & 0.04 & 1 & 3 & 0.09 & 3 \\
\hline 36 & Chrysomelidae sp. 2 & FI & 1 & 0.10 & 1 & 1 & 0.04 & 1 & 2 & 0.06 & 2 \\
\hline 37 & Chrysomelidae sp. 3 & FI & - & - & - & 1 & 0.04 & 1 & 1 & 0.03 & 1 \\
\hline
\end{tabular}

$A b d=$ abundancia, $\%=$ abundancia porcentual y $F r e c=$ frecuencia de captura en 20 muestras para cada estación y en 40 para el ensamblaje total. $\mathrm{DE}=$ depredadora, $\mathrm{CO}=$ coprófaga, $\mathrm{FI}=$ fitófaga, $\mathrm{MI}=$ micófaga, $\mathrm{NE}=$ necrófaga, $\mathrm{OM}=$ omnívora, $\mathrm{SA}=\mathrm{saprófaga}$, $\mathrm{IN}=$ indeterminada (desconocida), $\mathrm{CO} / \mathrm{NE}=$ copro-necrófaga, DE/MI= depredadora/micófaga, SA/MI: saprófaga/micófaga. 


\begin{tabular}{|c|c|c|c|c|c|c|c|c|c|c|c|}
\hline $\begin{array}{l}\text { Clave } \\
\text { - taxon }\end{array}$ & & $\begin{array}{l}\text { Hábitos } \\
\text { aliment. }\end{array}$ & & Lluvia & & & Secas & & & Total & \\
\hline 38 & $\begin{array}{l}\text { Chrysomelidae sp. } 4 \\
\text { CIIDAE }\end{array}$ & FI & - & - & - & 1 & 0.04 & 1 & 1 & 0.03 & 1 \\
\hline 39 & Ciidae sp. 1 & MI & 2 & 0.20 & 1 & - & - & - & 2 & 0.06 & 1 \\
\hline 40 & Ciidae sp. 2 & MI & - & - & - & 1 & 0.04 & 1 & 1 & 0.03 & 1 \\
\hline 41 & Ciidae sp. 3 & MI & - & - & - & 8 & 0.35 & 4 & 8 & 0.24 & 4 \\
\hline 42 & Ciidae sp. 4 & MI & 1 & 0.10 & 1 & 1 & 0.04 & 1 & 2 & 0.06 & 2 \\
\hline 43 & $\begin{array}{l}\text { Ciidae sp. } 5 \\
\text { CLERIDAE }\end{array}$ & MI & - & - & - & 3 & 0.13 & 3 & 3 & 0.09 & 3 \\
\hline 44 & Cleridae sp. 1 & $\mathrm{DE}$ & - & - & - & 1 & 0.04 & 1 & 1 & 0.03 & 1 \\
\hline 45 & $\begin{array}{l}\text { Cleridae sp. } 2 \\
\text { COLYDIIDAE }\end{array}$ & $\mathrm{DE}$ & - & - & - & 1 & 0.04 & 1 & 1 & 0.03 & 1 \\
\hline 46 & Colydiidae sp. 1 & MI & - & - & - & 2 & 0.09 & 2 & 2 & 0.06 & 2 \\
\hline 47 & Colydiidae sp. 2 & MI & - & - & - & 6 & 0.26 & 4 & 6 & 0.18 & 4 \\
\hline 48 & Colydiidae sp. 3 & MI & - & - & - & 1 & 0.04 & 1 & 1 & 0.03 & 1 \\
\hline 49 & Colydiidae sp. 4 & MI & 2 & 0.20 & 2 & - & - & - & 2 & 0.06 & 2 \\
\hline 50 & Colydiidae sp. 5 & MI & 3 & 0.29 & 3 & - & - & - & 3 & 0.09 & 3 \\
\hline 51 & Colydiidae sp. 6 & MI & - & - & - & 2 & 0.09 & 2 & 2 & 0.06 & 2 \\
\hline 52 & Colydiidae sp. 7 & MI & 1 & 0.10 & 1 & 1 & 0.04 & 1 & 2 & 0.06 & 2 \\
\hline 53 & $\begin{array}{l}\text { Colydiidae sp. } 8 \\
\text { CORYLOPHIDAE }\end{array}$ & MI & 1 & 0.10 & 1 & - & - & - & 1 & 0.03 & 1 \\
\hline 54 & Corylophidae sp. 1 & MI & - & - & - & 1 & 0.04 & 1 & 1 & 0.03 & 1 \\
\hline 55 & $\begin{array}{l}\text { Corylophidae sp. } 2 \\
\text { CRYPTOPHAGIDAE }\end{array}$ & MI & - & - & - & 3 & 0.13 & 2 & 3 & 0.09 & 2 \\
\hline 56 & Cryptophagidae sp. 1 & $\mathrm{SA} / \mathrm{MI}$ & - & - & - & 2 & 0.09 & 2 & 2 & 0.06 & 2 \\
\hline 57 & $\begin{array}{l}\text { Cryptophagidae sp. } 2 \\
\text { CUCUJIDAE }\end{array}$ & SA/MI & - & - & - & 1 & 0.04 & 1 & 1 & 0.03 & 1 \\
\hline 58 & Cucujidae sp. 1 & SA & - & - & - & 12 & 0.52 & 7 & 12 & 0.36 & 7 \\
\hline 59 & $\begin{array}{l}\text { Cucujidae sp. } 2 \\
\text { CURCULIONIDAE }\end{array}$ & $\mathrm{SA}$ & - & - & - & 1 & 0.04 & 1 & 1 & 0.03 & 1 \\
\hline 60 & Platypodini sp. & FI & - & - & - & 2 & 0.09 & 2 & 2 & 0.06 & 2 \\
\hline 61 & Scolytinae sp. 1 & FI & - & - & - & 2 & 0.09 & 2 & 2 & 0.06 & 2 \\
\hline 62 & Scolytinae sp. 2 & FI & 8 & 0.79 & 7 & 12 & 0.52 & 9 & 20 & 0.60 & 16 \\
\hline 63 & Scolytinae sp. 3 & FI & 1 & 0.10 & 1 & 2 & 0.09 & 2 & 3 & 0.09 & 3 \\
\hline 64 & Scolytinae sp. 4 & FI & 6 & 0.59 & 4 & 13 & 0.57 & 8 & 19 & 0.57 & 12 \\
\hline 65 & Scolytinae sp. 5 & FI & 1 & 0.10 & 1 & 1 & 0.04 & 1 & 2 & 0.06 & 2 \\
\hline 66 & Scolytinae sp. 6 & FI & 3 & 0.29 & 3 & 10 & 0.44 & 7 & 13 & 0.39 & 10 \\
\hline 67 & Scolytinae sp. 7 & FI & 1 & 0.10 & 1 & 8 & 0.35 & 4 & 9 & 0.27 & 5 \\
\hline 68 & Scolytinae sp. 8 & FI & - & - & - & 5 & 0.22 & 3 & 5 & 0.15 & 3 \\
\hline 69 & Scolytinae sp. 9 & FI & - & - & - & 2 & 0.09 & 2 & 2 & 0.06 & 2 \\
\hline 70 & Scolytinae sp. 10 & FI & - & - & - & 5 & 0.22 & 1 & 5 & 0.15 & 1 \\
\hline 71 & Scolytinae sp. 11 & FI & 2 & 0.20 & 1 & 2 & 0.09 & 2 & 4 & 0.12 & 3 \\
\hline 72 & Curculionidae sp. 1 & FI & - & - & - & 1 & 0.04 & 1 & 1 & 0.03 & 1 \\
\hline 73 & Curculionidae sp. 2 & FI & 3 & 0.29 & 2 & 2 & 0.09 & 2 & 5 & 0.15 & 4 \\
\hline 74 & Curculionidae sp. 3 & FI & 19 & 1.87 & 9 & 6 & 0.26 & 6 & 25 & 0.76 & 15 \\
\hline 75 & Curculionidae sp. 4 & FI & - & - & - & 1 & 0.04 & 1 & 1 & 0.03 & 1 \\
\hline 76 & Curculionidae sp. 5 & FI & - & - & - & 2 & 0.09 & 2 & 2 & 0.06 & 2 \\
\hline 77 & Curculionidae sp. 6 & FI & - & - & - & 1 & 0.04 & 1 & 1 & 0.03 & 1 \\
\hline 78 & Curculionidae sp. 7 & FI & - & - & - & 4 & 0.17 & 3 & 4 & 0.12 & 3 \\
\hline 79 & Curculionidae sp. 8 & FI & 2 & 0.20 & 2 & 7 & 0.31 & 5 & 9 & 0.27 & 7 \\
\hline 80 & Curculionidae sp. 9 & FI & - & - & - & 1 & 0.04 & 1 & 1 & 0.03 & 1 \\
\hline 81 & Curculionidae sp. 10 & FI & - & - & - & 1 & 0.04 & 1 & 1 & 0.03 & 1 \\
\hline 82 & Curculionidae sp. 11 & FI & - & - & - & 2 & 0.09 & 2 & 2 & 0.06 & 2 \\
\hline 83 & Curculionidae sp. 12 & FI & - & - & - & 1 & 0.04 & 1 & 1 & 0.03 & 1 \\
\hline 84 & Curculionidae sp. 13 & FI & - & - & - & 4 & 0.17 & 3 & 4 & 0.12 & 3 \\
\hline 85 & Curculionidae sp. 14 & FI & 1 & 0.10 & 1 & - & - & - & 1 & 0.03 & 1 \\
\hline 86 & Curculionidae sp. 15 & FI & 2 & 0.20 & 2 & - & - & - & 2 & 0.06 & 2 \\
\hline
\end{tabular}




\begin{tabular}{|c|c|c|c|c|c|c|c|c|c|c|c|}
\hline $\begin{array}{l}\text { Clave } \\
\text { - taxon }\end{array}$ & & $\begin{array}{l}\text { Hábitos } \\
\text { aliment. }\end{array}$ & & Lluvias & & & Secas & & & Total & \\
\hline 87 & Curculionidae sp. 16 & FI & - & - & - & 1 & 0.04 & 1 & 1 & 0.03 & 1 \\
\hline 88 & Curculionidae sp. 17 & FI & - & - & - & 1 & 0.04 & 1 & 1 & 0.03 & 1 \\
\hline 89 & $\begin{array}{l}\text { Curculionidae sp. } 18 \\
\text { DYTISCIDAE }\end{array}$ & FI & 1 & 0.10 & 1 & 1 & 0.04 & 1 & 2 & 0.06 & 2 \\
\hline 90 & Copelatus sp. & OM & 12 & 1.18 & 8 & 1 & 0.04 & 1 & 13 & 0.39 & 9 \\
\hline 91 & Dytiscidae sp. 1 & $\mathrm{OM}$ & 3 & 0.29 & 2 & - & - & - & 3 & 0.09 & 2 \\
\hline 92 & Dytiscidae sp. 2 & $\mathrm{OM}$ & 2 & 0.20 & 1 & 2 & 0.09 & 2 & 4 & 0.12 & 3 \\
\hline 93 & $\begin{array}{l}\text { Dytiscidae sp. } 3 \\
\text { ELATERIDAE }\end{array}$ & $\mathrm{OM}$ & - & - & - & 1 & 0.04 & 1 & 1 & 0.03 & 1 \\
\hline 94 & Cebrioninae sp. & OM & - & - & - & 2 & 0.09 & 1 & 2 & 0.06 & 1 \\
\hline 95 & Elateridae sp. 1 & $\mathrm{OM}$ & 1 & 0.10 & 1 & - & - & - & 1 & 0.03 & 1 \\
\hline 96 & Elateridae sp. 2 & $\mathrm{OM}$ & 2 & 0.20 & 2 & 1 & 0.04 & 1 & 3 & 0.09 & 3 \\
\hline 97 & Elateridae sp. 3 & $\mathrm{OM}$ & 3 & 0.29 & 3 & 3 & 0.13 & 2 & 6 & 0.18 & 5 \\
\hline 98 & Elateridae sp. 4 & $\mathrm{OM}$ & 1 & 0.10 & 1 & - & - & - & 1 & 0.03 & 1 \\
\hline 99 & Elateridae sp. 5 & $\mathrm{OM}$ & - & - & - & 1 & 0.04 & 1 & 1 & 0.03 & 1 \\
\hline 100 & Elateridae sp. 6 & $\mathrm{OM}$ & 2 & 0.20 & 2 & 1 & 0.04 & 1 & 3 & 0.09 & 3 \\
\hline 101 & Elateridae sp. 7 & $\mathrm{OM}$ & 1 & 0.10 & 1 & 3 & 0.13 & 2 & 4 & 0.12 & 3 \\
\hline 102 & Elateridae sp. 8 & $\mathrm{OM}$ & 1 & 0.10 & 1 & 2 & 0.09 & 2 & 3 & 0.09 & 3 \\
\hline 103 & Elateridae sp. 9 & $\mathrm{OM}$ & 7 & 0.69 & 3 & 47 & 2.05 & 6 & 54 & 1.63 & 9 \\
\hline 104 & Elateridae sp. 10 & $\mathrm{OM}$ & - & - & - & 1 & 0.04 & 1 & 1 & 0.03 & 1 \\
\hline 105 & Elateridae sp. 11 & $\mathrm{OM}$ & - & - & - & 1 & 0.04 & 1 & 1 & 0.03 & 1 \\
\hline 106 & Elateridae sp. 12 & $\mathrm{OM}$ & 3 & 0.29 & 3 & - & - & - & 3 & 0.09 & 3 \\
\hline 107 & Elateridae sp. 13 & $\mathrm{OM}$ & - & - & - & 2 & 0.09 & 2 & 2 & 0.06 & 2 \\
\hline 108 & Elateridae sp. 14 & $\mathrm{OM}$ & - & - & - & 2 & 0.09 & 2 & 2 & 0.06 & 2 \\
\hline 109 & Elateridae sp. 15 & $\mathrm{OM}$ & 1 & 0.10 & 1 & - & - & - & 1 & 0.03 & 1 \\
\hline 110 & $\begin{array}{l}\text { Elateridae sp. } 16 \\
\text { ENDOMYCHIDAE }\end{array}$ & $\mathrm{OM}$ & - & - & - & 1 & 0.04 & 1 & 1 & 0.03 & 1 \\
\hline 111 & $\begin{array}{l}\text { Endomychidae sp. } \\
\text { EROTYLIDAE }\end{array}$ & MI & - & - & - & 1 & 0.04 & 1 & 1 & 0.03 & 1 \\
\hline 112 & Erotylidae sp. 1 & MI & 1 & 0.10 & 1 & - & - & - & 1 & 0.03 & 1 \\
\hline 113 & Erotylidae sp. 2 & MI & - & - & - & 1 & 0.04 & 1 & 1 & 0.03 & 1 \\
\hline 114 & $\begin{array}{l}\text { Erotylidae sp. } 3 \\
\text { HYDROPHILIDAE }\end{array}$ & MI & - & - & - & 1 & 0.04 & 1 & 1 & 0.03 & 1 \\
\hline 115 & Sphaeridiinae sp. 1 & $\mathrm{OM}$ & 44 & 4.32 & 13 & 24 & 1.05 & 7 & 68 & 2.06 & 20 \\
\hline 116 & Sphaeridiinae sp. 2 & $\mathrm{OM}$ & 5 & 0.49 & 4 & 8 & 0.35 & 5 & 13 & 0.39 & 9 \\
\hline 117 & Sphaeridiinae sp. 3 & $\mathrm{OM}$ & 1 & 0.10 & 1 & 1 & 0.04 & 1 & 2 & 0.06 & 2 \\
\hline 118 & Hydrophilidae sp. 1 & $\mathrm{OM}$ & 5 & 0.49 & 2 & - & - & - & 5 & 0.15 & 2 \\
\hline 119 & $\begin{array}{l}\text { Hydrophilidae sp. } 2 \\
\text { HISTERIDAE }\end{array}$ & $\mathrm{OM}$ & 1 & 0.10 & 1 & - & - & - & 1 & 0.03 & 1 \\
\hline 120 & Hololepta sp. & $\mathrm{DE}$ & - & - & - & 1 & 0.04 & 1 & 1 & 0.03 & 1 \\
\hline 121 & Histeridae sp. 1 & $\mathrm{DE}$ & 14 & 1.38 & 6 & 34 & 1.48 & 6 & 48 & 1.45 & 12 \\
\hline 122 & Histeridae sp. 2 & $\mathrm{DE}$ & 2 & 0.20 & 2 & 4 & 0.17 & 4 & 6 & 0.18 & 6 \\
\hline 123 & Histeridae sp. 3 & $\mathrm{DE}$ & - & - & - & 7 & 0.31 & 3 & 7 & 0.21 & 3 \\
\hline 124 & Histeridae sp. 4 & $\mathrm{DE}$ & - & - & - & 1 & 0.04 & 1 & 1 & 0.03 & 1 \\
\hline 125 & Histeridae sp. 5 & $\mathrm{DE}$ & - & - & - & 1 & 0.04 & 1 & 1 & 0.03 & 1 \\
\hline 126 & Histeridae sp. 6 & $\mathrm{DE}$ & - & - & - & 1 & 0.04 & 1 & 1 & 0.03 & 1 \\
\hline 127 & Histeridae sp. 7 & $\mathrm{DE}$ & 1 & 0.10 & 1 & - & - & - & 1 & 0.03 & 1 \\
\hline 128 & $\begin{array}{l}\text { Histeridae sp. } 8 \\
\text { LAMPYRIDAE }\end{array}$ & $\mathrm{DE}$ & - & - & - & 4 & 0.17 & 3 & 4 & 0.12 & 3 \\
\hline 129 & $\begin{array}{l}\text { Photinus cangruus } \\
\text { LATRIIDIDAE }\end{array}$ & $\mathrm{DE}$ & - & - & - & 8 & 0.35 & 5 & 8 & 0.24 & 5 \\
\hline 130 & Latriididae sp. 1 & MI & - & - & - & 3 & 0.13 & 3 & 3 & 0.09 & 3 \\
\hline 131 & $\begin{array}{l}\text { Latriididae sp. } 2 \\
\text { LEIODIDAE }\end{array}$ & MI & - & - & - & 10 & 0.44 & 7 & 10 & 0.30 & 7 \\
\hline 132 & Leiodes sp. 1 & MI & 1 & 0.10 & 1 & 2 & 0.09 & 1 & 3 & 0.09 & 2 \\
\hline 133 & Leiodes sp. 2 & MI & - & - & - & 2 & 0.09 & 2 & 2 & 0.06 & 2 \\
\hline
\end{tabular}




\begin{tabular}{|c|c|c|c|c|c|c|c|c|c|c|c|}
\hline $\begin{array}{l}\text { Clave } \\
\text { - taxon }\end{array}$ & & $\begin{array}{l}\text { Hábitos } \\
\text { aliment. }\end{array}$ & & Lluvias & & & Secas & & & Total & \\
\hline 134 & Leiodinae sp. 1 & MI & 2 & 0.20 & 2 & 12 & 0.52 & 9 & 14 & 0.42 & 11 \\
\hline 135 & Leiodinae sp. 2 & MI & - & - & - & 1 & 0.04 & 1 & 1 & 0.03 & 1 \\
\hline 136 & Ecarinoespherula sp. & MI & 6 & 0.59 & 5 & 29 & 1.27 & 14 & 35 & 1.06 & 19 \\
\hline 137 & Agatidium sp. & MI & - & - & - & 2 & 0.09 & 2 & 2 & 0.06 & 2 \\
\hline 138 & Anisotoma sp. 1 & MI & 1 & 0.10 & 1 & 12 & 0.52 & 6 & 13 & 0.39 & 7 \\
\hline 139 & Anisotoma sp. 2 & MI & 1 & 0.10 & 1 & 7 & 0.31 & 5 & 8 & 0.24 & 6 \\
\hline 140 & Ptomophagus (Adelops) & $\mathrm{NE}$ & - & - & - & 1 & 0.04 & 1 & 1 & 0.03 & 1 \\
\hline 141 & $\begin{array}{l}\text { sp. } 1 \\
\text { Ptomophagus (Adelops) }\end{array}$ & NE & 1 & 0.10 & 1 & 4 & 0.17 & 4 & 5 & 0.15 & 5 \\
\hline 142 & sp. 2 Ptomophagus (Adelops) & $\mathrm{NE}$ & - & - & - & 1 & 0.04 & 1 & 1 & 0.03 & 1 \\
\hline 143 & Sp. 3 issochaetus sp. & $\mathrm{NE}$ & - & - & - & 2 & 0.09 & 2 & 2 & 0.06 & 2 \\
\hline 144 & Nemadus sp. & $\mathrm{NE}$ & 8 & 0.79 & 5 & 13 & 0.57 & 8 & 21 & 0.63 & 13 \\
\hline 145 & $\begin{array}{l}\text { Cholevinae? sp. } \\
\text { LUTROCHIDAE }\end{array}$ & $\mathrm{NE}$ & 2 & 0.20 & 2 & 10 & 0.44 & 9 & 12 & 0.36 & 11 \\
\hline 146 & $\begin{array}{l}\text { Lutrochus? sp. } \\
\text { LYCIDAE }\end{array}$ & FI & - & - & - & 3 & 0.13 & 2 & 3 & 0.09 & 2 \\
\hline 147 & Lycidae sp. 1 & $\mathrm{DE}$ & - & - & - & 4 & 0.17 & 4 & 4 & 0.12 & 4 \\
\hline 148 & Lycidae sp. 2 & DE & 3 & 0.29 & 3 & - & - & - & 3 & 0.09 & 3 \\
\hline 149 & $\begin{array}{l}\text { Lycidae sp. } 3 \\
\text { MELANDRYIDAE }\end{array}$ & $\mathrm{DE}$ & - & - & - & 1 & 0.04 & 1 & 1 & 0.03 & 1 \\
\hline 150 & Melandryidae sp. 1 & $\mathrm{OM}$ & - & - & - & 1 & 0.04 & 1 & 1 & 0.03 & 1 \\
\hline 151 & Melandryidae sp. 2 & $\mathrm{OM}$ & - & - & - & 3 & 0.13 & 2 & 3 & 0.09 & 2 \\
\hline 152 & $\begin{array}{l}\text { Melandryidae sp. } 3 \\
\text { MORDELLIDAE }\end{array}$ & $\mathrm{OM}$ & - & - & - & 1 & 0.04 & 1 & 1 & 0.03 & 1 \\
\hline 153 & Mordellidae sp. 1 & FI & - & - & - & 6 & 0.26 & 3 & 6 & 0.18 & 3 \\
\hline 154 & $\begin{array}{l}\text { Mordellidae sp. } 2 \\
\text { MYCETOPHAGIDAE }\end{array}$ & FI & 1 & 0.10 & 1 & 1 & 0.04 & 1 & 2 & 0.06 & 2 \\
\hline 155 & Mycetophagidae sp. 1 & MI & - & - & - & 1 & 0.04 & 1 & 1 & 0.03 & 1 \\
\hline 156 & $\begin{array}{l}\text { Mycetophagidae sp. } 2 \\
\text { NITIDULIDAE }\end{array}$ & MI & - & - & - & 9 & 0.39 & 2 & 9 & 0.27 & 2 \\
\hline 157 & Carpophilus sp. & SA/MI & - & - & - & 1 & 0.04 & 1 & 1 & 0.03 & 1 \\
\hline 158 & Carpophilinae sp. 1 & SA/MI & - & - & - & 1 & 0.04 & 1 & 1 & 0.03 & 1 \\
\hline 159 & Carpophilinae sp. 2 & SA/MI & - & - & - & 2 & 0.09 & 2 & 2 & 0.06 & 2 \\
\hline 160 & Carpophilinae sp. 3 & SA/MI & - & - & - & 1 & 0.04 & 1 & 1 & 0.03 & 1 \\
\hline 161 & Nitidula sp. 1 & SA/MI & 27 & 2.65 & 10 & 58 & 2.53 & 14 & 85 & 2.57 & 24 \\
\hline 162 & Nitidula sp. 2 & SA/MI & - & - & - & 2 & 0.09 & 2 & 2 & 0.06 & 2 \\
\hline 163 & Nitidula sp. 3 & SA/MI & - & - & - & 1 & 0.04 & 1 & 1 & 0.03 & 1 \\
\hline 164 & Thalycra sp. 1 & SA/MI & 142 & 13.95 & 18 & 25 & 1.09 & 5 & 167 & 5.05 & 23 \\
\hline 165 & Thalycra sp. 2 & SA/MI & 3 & 0.29 & 2 & 1 & 0.04 & 1 & 4 & 0.12 & 3 \\
\hline 166 & Thalycra sp. 3 & SA/MI & 11 & 1.08 & 5 & 32 & 1.40 & 7 & 43 & 1.30 & 12 \\
\hline 167 & Thalycra sp. 4 & SA/MI & 1 & 0.10 & 1 & - & - & - & 1 & 0.03 & 1 \\
\hline 168 & Nitidulinae sp. & SA/MI & 12 & 1.18 & 8 & 8 & 0.35 & 7 & 20 & 0.60 & 15 \\
\hline 169 & Nitidulidae sp. 1 & SA/MI & - & - & - & 4 & 0.17 & 3 & 4 & 0.12 & 3 \\
\hline 170 & Nitidulidae sp. 2 & SA/MI & - & - & - & 1 & 0.04 & 1 & 1 & 0.03 & 1 \\
\hline 171 & Nitidulidae sp. 3 & SA/MI & - & - & - & 1 & 0.04 & 1 & 1 & 0.03 & 1 \\
\hline 172 & Nitidulidae sp. 4 & SA/MI & 2 & 0.20 & 2 & 20 & 0.87 & 10 & 22 & 0.67 & 12 \\
\hline 173 & Nitidulidae sp. 5 & SA/MI & 2 & 0.20 & 2 & - & - & - & 2 & 0.06 & 2 \\
\hline 174 & Nitidulidae sp. 6 & SA/MI & 2 & 0.20 & 2 & 4 & 0.17 & 3 & 6 & 0.18 & 5 \\
\hline 175 & Nitidulidae sp. 7 & SA/MI & - & - & - & 1 & 0.04 & 1 & 1 & 0.03 & 1 \\
\hline 176 & $\begin{array}{l}\text { Nitidulidae sp. } 8 \\
\text { NOSODENDRIDAE }\end{array}$ & SA/MI & 1 & 0.10 & 1 & - & - & - & 1 & 0.03 & 1 \\
\hline 177 & Nosodendron sp. & $\mathrm{DE}$ & 7 & 0.69 & 5 & 1 & 0.04 & 1 & 8 & 0.24 & 6 \\
\hline 178 & $\begin{array}{l}\text { Nosodendridae sp. } \\
\text { NOTHERIDAE }\end{array}$ & $\mathrm{DE}$ & - & - & - & 1 & 0.04 & 1 & 1 & 0.03 & 1 \\
\hline 179 & $\begin{array}{l}\text { Notheridae sp. } \\
\text { PHENGODIDAE }\end{array}$ & $\mathrm{DE}$ & - & - & - & 1 & 0.04 & 1 & 1 & 0.03 & 1 \\
\hline
\end{tabular}




\begin{tabular}{|c|c|c|c|c|c|c|c|c|c|c|c|}
\hline $\begin{array}{l}\text { Clave } \\
\text { - taxon }\end{array}$ & & $\begin{array}{l}\text { Hábitos } \\
\text { aliment. }\end{array}$ & & Lluvias & & & Secas & & & Total & \\
\hline 180 & Cenophengus minizi & DE & - & - & - & 1 & 0.04 & 1 & 1 & 0.03 & 1 \\
\hline 181 & Phengodes sp. & DE & - & - & - & 1 & 0.04 & 1 & 1 & 0.03 & 1 \\
\hline 182 & $\begin{array}{l}\text { Taximastinocerus bruneus } \\
\text { PTIILIDAE }\end{array}$ & $\mathrm{DE}$ & - & - & - & 22 & 0.96 & 8 & 22 & 0.67 & 8 \\
\hline 183 & $\begin{array}{l}\text { Ptiilidae sp. } \\
\text { PTILODACTYLIDAE }\end{array}$ & MI & - & - & - & 21 & 0.92 & 6 & 21 & 0.63 & 6 \\
\hline 184 & Ptilodactyla sp. 1 & IN & - & - & - & 2 & 0.09 & 2 & 2 & 0.06 & 2 \\
\hline 185 & Ptilodactyla sp. 2 & IN & 2 & 0.20 & 2 & 4 & 0.17 & 4 & 6 & 0.18 & 6 \\
\hline 186 & Ptilodactyla sp. 3 & IN & 2 & 0.20 & 2 & 1 & 0.04 & 1 & 3 & 0.09 & 3 \\
\hline 187 & $\begin{array}{l}\text { Ptilodactylidae sp. } \\
\text { PYROCHROIDAE }\end{array}$ & IN & - & - & - & 1 & 0.04 & 1 & 1 & 0.03 & 1 \\
\hline 188 & Pedilus sp. & IN & 1 & 0.10 & 1 & 1 & 0.04 & 1 & 2 & 0.06 & 2 \\
\hline 189 & Pyrochroidae sp. 1 & IN & - & - & - & 1 & 0.04 & 1 & 1 & 0.03 & 1 \\
\hline 190 & $\begin{array}{l}\text { Pyrochroidae sp. } 2 \\
\text { RHIPICERIDAE }\end{array}$ & IN & - & - & - & 1 & 0.04 & 1 & 1 & 0.03 & 1 \\
\hline 191 & Sandalus? sp. 1 & IN & 3 & 0.29 & 3 & - & - & - & 3 & 0.09 & 3 \\
\hline 192 & $\begin{array}{l}\text { Sandalus? sp. } 2 \\
\text { SCARABAEIDAE }\end{array}$ & IN & 1 & 0.10 & 1 & 1 & 0.04 & 1 & 2 & 0.06 & 2 \\
\hline 193 & Anomala sp. & FI & - & - & - & 1 & 0.04 & 1 & 1 & 0.03 & 1 \\
\hline 194 & Aphodinae sp. 1 & $\mathrm{CO}$ & 25 & 2.46 & 10 & 5 & 0.22 & 5 & 30 & 0.91 & 15 \\
\hline 195 & Aphodinae sp. 2 & $\mathrm{CO}$ & - & - & - & 2 & 0.09 & 1 & 2 & 0.06 & 1 \\
\hline 196 & Coprophanaeus gilli & $\mathrm{CO} / \mathrm{NE}$ & 3 & 0.29 & 1 & - & - & - & 3 & 0.09 & 1 \\
\hline 197 & Deltochilum mexicanum & $\mathrm{NE}$ & 43 & 4.22 & 16 & 1 & 0.04 & 1 & 44 & 1.33 & 17 \\
\hline 198 & Dichotomius satanas & $\mathrm{CO}$ & 29 & 2.85 & 11 & 1 & 0.04 & 1 & 30 & 0.91 & 12 \\
\hline 199 & Ontherus mexicanus & $\mathrm{CO}$ & 56 & 5.50 & 14 & 4 & 0.17 & 3 & 60 & 1.81 & 17 \\
\hline 200 & Onthophagus sp. & $\mathrm{CO}$ & 5 & 0.49 & 2 & - & - & - & 5 & 0.15 & 2 \\
\hline 201 & Phanaeus amethystinus & $\mathrm{CO}$ & 1 & 0.10 & 1 & - & - & - & 1 & 0.03 & 1 \\
\hline 202 & Phyllophaga (Ph.) atra & FI & 6 & 0.59 & 4 & 2 & 0.09 & 2 & 8 & 0.24 & 6 \\
\hline 203 & Phyllophaga nr. scissa & FI & 7 & 0.69 & 6 & 4 & 0.17 & 3 & 11 & 0.33 & 9 \\
\hline 204 & Phyllophaga sp. 1 & FI & 2 & 0.20 & 2 & - & - & - & 2 & 0.06 & 2 \\
\hline 205 & Phyllophaga sp. 2 & FI & 1 & 0.10 & 1 & 4 & 0.17 & 2 & 5 & 0.15 & 3 \\
\hline 206 & Phyllophaga sp. 3 & FI & 2 & 0.20 & 2 & 7 & 0.31 & 4 & 9 & 0.27 & 6 \\
\hline 207 & $\begin{array}{l}\text { Phyllophaga sp. } 4 \\
\text { SCIRTIDAE }\end{array}$ & FI & - & - & - & 9 & 0.39 & 6 & 9 & 0.27 & 6 \\
\hline 208 & $\begin{array}{l}\text { Scirtidae sp. } \\
\text { SILPHIDAE }\end{array}$ & IN & 1 & 0.10 & 1 & 26 & 1.14 & 6 & 27 & 0.82 & 7 \\
\hline 209 & $\begin{array}{l}\text { Nicrophorus olidus } \\
\text { SPHINDIDAE }\end{array}$ & $\mathrm{NE}$ & 39 & 3.83 & 8 & 23 & 1.00 & 6 & 62 & 1.87 & 14 \\
\hline 210 & Eurysphindus sp. & MI & - & - & - & 2 & 0.09 & 2 & 2 & 0.06 & 2 \\
\hline 211 & Carinisphindus sp. & MI & - & - & - & 3 & 0.13 & 2 & 3 & 0.09 & 2 \\
\hline 212 & $\begin{array}{l}\text { Sphindidae sp. } \\
\text { STAPHILINIDAE }\end{array}$ & MI & - & - & - & 1 & 0.04 & 1 & 1 & 0.03 & 1 \\
\hline 213 & Aleocharinae sp. 1 & $\mathrm{DE}$ & 10 & 0.98 & 6 & 25 & 1.09 & 7 & 35 & 1.06 & 13 \\
\hline 214 & Aleocharinae sp. 2 & $\mathrm{DE}$ & 7 & 0.69 & 6 & 10 & 0.44 & 6 & 17 & 0.51 & 12 \\
\hline 215 & Aleocharinae sp. 3 & $\mathrm{DE}$ & 2 & 0.20 & 2 & 22 & 0.96 & 10 & 24 & 0.73 & 12 \\
\hline 216 & Aleocharinae sp. 4 & DE & 2 & 0.20 & 2 & 3 & 0.13 & 2 & 5 & 0.15 & 4 \\
\hline 217 & Aleocharinae sp. 5 & $\mathrm{DE}$ & 2 & 0.20 & 2 & 14 & 0.61 & 4 & 16 & 0.48 & 6 \\
\hline 218 & Aleocharinae sp. 6 & $\mathrm{DE}$ & 1 & 0.10 & 1 & - & - & - & 1 & 0.03 & 1 \\
\hline 219 & Aleocharinae sp. 7 & $\mathrm{DE}$ & 17 & 1.67 & 5 & 4 & 0.17 & 3 & 21 & 0.63 & 8 \\
\hline 220 & Aleocharinae sp. 8 & $\mathrm{DE}$ & 96 & 9.43 & 13 & 307 & 13.41 & 19 & 403 & 12.18 & 32 \\
\hline 221 & Aleocharinae sp. 9 & $\mathrm{DE}$ & - & - & - & 1 & 0.04 & 1 & 1 & 0.03 & 1 \\
\hline 222 & Aleocharinae sp. 10 & $\mathrm{DE}$ & - & - & - & 4 & 0.17 & 4 & 4 & 0.12 & 4 \\
\hline 223 & Aleocharinae sp. 11 & $\mathrm{DE}$ & - & - & - & 5 & 0.22 & 5 & 5 & 0.15 & 5 \\
\hline 224 & Aleocharinae sp. 12 & $\mathrm{DE}$ & 1 & 0.10 & 1 & - & - & - & 1 & 0.03 & 1 \\
\hline 225 & Aleocharinae sp. 13 & $\mathrm{DE}$ & - & - & - & 1 & 0.04 & 1 & 1 & 0.03 & 1 \\
\hline 226 & Aleocharinae sp. 14 & $\mathrm{DE}$ & - & - & - & 2 & 0.09 & 2 & 2 & 0.06 & 2 \\
\hline
\end{tabular}




\begin{tabular}{|c|c|c|c|c|c|c|c|c|c|c|c|}
\hline $\begin{array}{l}\text { Clave } \\
\text { - taxon }\end{array}$ & & $\begin{array}{l}\text { Hábitos } \\
\text { aliment. }\end{array}$ & & Lluvias & & & Secas & & & Total & \\
\hline 227 & Aleocharinae sp. 15 & DE & - & - & - & 28 & 1.22 & 6 & 28 & 0.85 & 6 \\
\hline 228 & Aleocharinae sp. 16 & $\mathrm{DE}$ & 1 & 0.10 & 1 & 14 & 0.61 & 8 & 15 & 0.45 & 9 \\
\hline 229 & Aleocharinae sp. 17 & $\mathrm{DE}$ & 5 & 0.49 & 3 & 1 & 0.04 & 1 & 6 & 0.18 & 4 \\
\hline 230 & Aleocharinae sp. 18 & $\mathrm{DE}$ & 3 & 0.29 & 2 & 2 & 0.09 & 2 & 5 & 0.15 & 4 \\
\hline 231 & Aleocharinae sp. 19 & DE & - & - & - & 11 & 0.48 & 3 & 11 & 0.33 & 3 \\
\hline 232 & Aleocharinae sp. 20 & DE & 6 & 0.59 & 5 & 54 & 2.36 & 11 & 60 & 1.81 & 16 \\
\hline 233 & Aleocharinae sp. 21 & $\mathrm{DE}$ & 1 & 0.10 & 1 & 7 & 0.31 & 4 & 8 & 0.24 & 5 \\
\hline 234 & Aleocharinae sp. 22 & DE & - & - & - & 4 & 0.17 & 3 & 4 & 0.12 & 3 \\
\hline 235 & Aleocharinae sp. 23 & DE & - & - & - & 3 & 0.13 & 3 & 3 & 0.09 & 3 \\
\hline 236 & Aleocharinae sp. 24 & DE & 1 & 0.10 & 1 & 20 & 0.87 & 7 & 21 & 0.63 & 8 \\
\hline 237 & Aleocharinae sp. 25 & $\mathrm{DE}$ & 9 & 0.88 & 3 & 17 & 0.74 & 8 & 26 & 0.79 & 11 \\
\hline 238 & Aleocharinae sp. 26 & DE & 2 & 0.20 & 1 & - & - & - & 2 & 0.06 & 1 \\
\hline 239 & Aleocharinae sp. 27 & $\mathrm{DE}$ & 3 & 0.29 & 2 & 18 & 0.79 & 7 & 21 & 0.63 & 9 \\
\hline 240 & Aleocharinae sp. 28 & DE & 1 & 0.10 & 1 & 2 & 0.09 & 2 & 3 & 0.09 & 3 \\
\hline 241 & Aleocharinae sp. 29 & $\mathrm{DE}$ & - & - & - & 4 & 0.17 & 4 & 4 & 0.12 & 4 \\
\hline 242 & Aleocharinae sp. 30 & $\mathrm{DE}$ & 2 & 0.20 & 1 & 2 & 0.09 & 1 & 4 & 0.12 & 2 \\
\hline 243 & Aleocharinae sp. 31 & DE & - & - & - & 1 & 0.04 & 1 & 1 & 0.03 & 1 \\
\hline 244 & Aleocharinae sp. 32 & DE & 1 & 0.10 & 1 & 2 & 0.09 & 2 & 3 & 0.09 & 3 \\
\hline 245 & Aleocharinae sp. 33 & $\mathrm{DE}$ & - & - & - & 1 & 0.04 & 1 & 1 & 0.03 & 1 \\
\hline 246 & Aleocharinae sp. 34 & $\mathrm{DE}$ & - & - & - & 2 & 0.09 & 2 & 2 & 0.06 & 2 \\
\hline 247 & Aleocharinae sp. 35 & DE & 1 & 0.10 & 1 & 2 & 0.09 & 2 & 3 & 0.09 & 3 \\
\hline 248 & Aleocharinae sp. 36 & DE & 1 & 0.10 & 1 & 3 & 0.13 & 3 & 4 & 0.12 & 4 \\
\hline 249 & Aleocharinae sp. 37 & $\mathrm{DE}$ & 1 & 0.10 & 1 & - & - & - & 1 & 0.03 & 1 \\
\hline 250 & Aleocharinae sp. 38 & $\mathrm{DE}$ & - & - & - & 4 & 0.17 & 3 & 4 & 0.12 & 3 \\
\hline 251 & Aleocharinae sp. 39 & $\mathrm{DE}$ & - & - & - & 4 & 0.17 & 4 & 4 & 0.12 & 4 \\
\hline 252 & Aleocharinae sp. 40 & $\mathrm{DE}$ & - & - & - & 2 & 0.09 & 2 & 2 & 0.06 & 2 \\
\hline 253 & Aleocharinae sp. 41 & $\mathrm{DE}$ & - & - & - & 3 & 0.13 & 3 & 3 & 0.09 & 3 \\
\hline 254 & Aleocharinae sp. 42 & $\mathrm{DE}$ & - & - & - & 1 & 0.04 & 1 & 1 & 0.03 & 1 \\
\hline 255 & Aleocharinae sp. 43 & DE & 1 & 0.10 & 1 & 1 & 0.04 & 1 & 2 & 0.06 & 2 \\
\hline 256 & Aleocharinae sp. 44 & $\mathrm{DE}$ & 1 & 0.10 & 1 & - & - & - & 1 & 0.03 & 1 \\
\hline 257 & Aleocharinae sp. 45 & $\mathrm{DE}$ & - & - & - & 1 & 0.04 & 1 & 1 & 0.03 & 1 \\
\hline 258 & Anotylus sp. 1 & SA & 9 & 0.88 & 4 & 27 & 1.18 & 13 & 36 & 1.09 & 17 \\
\hline 259 & Anotylus sp. 2 & SA & 5 & 0.49 & 4 & 6 & 0.26 & 6 & 11 & 0.33 & 10 \\
\hline 260 & Anotylus sp. 3 & SA & - & - & - & 2 & 0.09 & 2 & 2 & 0.06 & 2 \\
\hline 261 & Anotylus sp. 4 & SA & 2 & 0.20 & 2 & - & - & - & 2 & 0.06 & 2 \\
\hline 262 & Anotylus sp. 5 & SA & - & - & - & 1 & 0.04 & 1 & 1 & 0.03 & 1 \\
\hline 263 & Baeocera sp. 1 & MI & - & - & - & 4 & 0.17 & 3 & 4 & 0.12 & 3 \\
\hline 264 & Baeocera sp. 2 & MI & - & - & - & 3 & 0.13 & 2 & 3 & 0.09 & 2 \\
\hline 265 & Baeocera sp. 3 & MI & - & - & - & 1 & 0.04 & 1 & 1 & 0.03 & 1 \\
\hline 266 & Belonuchus alternans & $\mathrm{DE}$ & 19 & 1.87 & 9 & 28 & 1.22 & 9 & 47 & 1.42 & 18 \\
\hline 267 & Belonuchus colon & DE & 5 & 0.49 & 3 & 13 & 0.57 & 8 & 18 & 0.54 & 11 \\
\hline 268 & Belonuchus sp. 1 & $\mathrm{DE}$ & - & - & - & 22 & 0.96 & 3 & 22 & 0.67 & 3 \\
\hline 269 & Belonuchus sp. 2 & DE & - & - & - & 1 & 0.04 & 1 & 1 & 0.03 & 1 \\
\hline 270 & Belonuchus sp. 3 & $\mathrm{DE}$ & - & - & - & 1 & 0.04 & 1 & 1 & 0.03 & 1 \\
\hline 271 & Bryoporus sp. & MI & 3 & 0.29 & 3 & 20 & 0.87 & 10 & 23 & 0.70 & 13 \\
\hline 272 & Chroaptomus flagrans & DE & - & - & - & 2 & 0.09 & 2 & 2 & 0.06 & 2 \\
\hline 273 & Coproporus sp. & SA & - & - & - & 6 & 0.26 & 3 & 6 & 0.18 & 3 \\
\hline 274 & Cyparium terminale & MI & 1 & 0.10 & 1 & - & - & - & 1 & 0.03 & 1 \\
\hline 275 & Eleusis sp. 1 & SA & - & - & - & 1 & 0.04 & 1 & 1 & 0.03 & 1 \\
\hline 276 & Eleusis sp. 2 & SA & - & - & - & 1 & 0.04 & 1 & 1 & 0.03 & 1 \\
\hline 277 & Eustilicus sp. 1 & $\mathrm{DE}$ & - & - & - & 9 & 0.39 & 5 & 9 & 0.27 & 5 \\
\hline 278 & Gabrius sp. 1 & $\mathrm{DE}$ & - & - & - & 6 & 0.26 & 6 & 6 & 0.18 & 6 \\
\hline 279 & Heterothops sp. & $\mathrm{DE}$ & - & - & - & 41 & 1.79 & 15 & 41 & 1.24 & 15 \\
\hline 280 & Hypotelus sp. & SA & - & - & - & 1 & 0.04 & 1 & 1 & 0.03 & 1 \\
\hline 281 & Ischnosoma sp. & MI & - & - & - & 2 & 0.09 & 2 & 2 & 0.06 & 2 \\
\hline 282 & Leptochirus sp. & SA & 1 & 0.10 & 1 & 1 & 0.04 & 1 & 2 & 0.06 & 2 \\
\hline
\end{tabular}




\begin{tabular}{|c|c|c|c|c|c|c|c|c|c|c|c|}
\hline $\begin{array}{l}\text { Clave } \\
\text { - taxon }\end{array}$ & & $\begin{array}{l}\text { Hábitos } \\
\text { aliment. }\end{array}$ & & Lluvias & & & Secas & & & Total & \\
\hline 283 & Lispinus sp. & SA & 2 & 0.20 & 2 & 5 & 0.22 & 3 & 7 & 0.21 & 5 \\
\hline 284 & Litocharodes sp. & $\mathrm{DE}$ & - & - & - & 1 & 0.04 & 1 & 1 & 0.03 & 1 \\
\hline 285 & Lordithon antenatus & MI & 2 & 0.20 & 2 & 1 & 0.04 & 1 & 3 & 0.09 & 3 \\
\hline 286 & Lordithon nubicola & MI & 4 & 0.39 & 2 & 21 & 0.92 & 8 & 25 & 0.76 & 10 \\
\hline 287 & Megalopinus sp & DE & - & - & - & 1 & 0.04 & 1 & 1 & 0.03 & 1 \\
\hline 288 & Misantlius carinulatus & DE & 2 & 0.20 & 2 & - & - & - & 2 & 0.06 & 2 \\
\hline 289 & Neohypnus sp. 1 & DE & 8 & 0.79 & 5 & 32 & 1.40 & 10 & 40 & 1.21 & 15 \\
\hline 290 & Neohypnus sp. 2 & $\mathrm{DE}$ & 1 & 0.10 & 1 & - & - & - & 1 & 0.03 & 1 \\
\hline 291 & Oligotergus sp. & DE & 13 & 1.28 & 7 & 20 & 0.87 & 7 & 33 & 1.00 & 14 \\
\hline 292 & Omaliinae sp. 1 & DE & - & - & - & 4 & 0.17 & 2 & 4 & 0.12 & 2 \\
\hline 293 & Omaliinae sp. 2 & $\mathrm{DE}$ & 1 & 0.10 & 1 & 1 & 0.04 & 1 & 2 & 0.06 & 2 \\
\hline 294 & Oxytelus sp. 1 & SA & - & - & - & 1 & 0.04 & 1 & 1 & 0.03 & 1 \\
\hline 295 & Oxytelinae sp. 1 & SA & - & - & - & 3 & 0.13 & 2 & 3 & 0.09 & 2 \\
\hline 296 & Paederomimus sp. 1 & $\mathrm{DE}$ & 1 & 0.10 & 1 & 3 & 0.13 & 3 & 4 & 0.12 & 4 \\
\hline 297 & Paederomimus sp. 2 & $\mathrm{DE}$ & - & - & - & 1 & 0.04 & 1 & 1 & 0.03 & 1 \\
\hline 298 & Palaminus sp. 1 & DE & - & - & - & 1 & 0.04 & 1 & 1 & 0.03 & 1 \\
\hline 299 & Philonthus sp. 1 & DE & 3 & 0.29 & 2 & 88 & 3.84 & 7 & 91 & 2.75 & 9 \\
\hline 300 & Philonthus sp. 2 & DE & 6 & 0.59 & 2 & 11 & 0.48 & 4 & 17 & 0.51 & 6 \\
\hline 301 & Philonthus sp. 3 & $\mathrm{DE}$ & 7 & 0.69 & 2 & 46 & 2.01 & 13 & 53 & 1.60 & 15 \\
\hline 302 & Philonthus sp. 4 & $\mathrm{DE}$ & 1 & 0.10 & 1 & 1 & 0.04 & 1 & 2 & 0.06 & 2 \\
\hline 303 & Platydracus & $\mathrm{DE}$ & 3 & 0.29 & 1 & 31 & 1.35 & 10 & 34 & 1.03 & 11 \\
\hline 304 & $\begin{array}{l}\text { fuscomaculatus } \\
\text { Priochirus sp. }\end{array}$ & SA & - & - & - & 6 & 0.26 & 4 & 6 & 0.18 & 4 \\
\hline 305 & Pselaphinae sp. 1 & $\mathrm{DE}$ & 3 & 0.29 & 2 & 3 & 0.13 & 3 & 6 & 0.18 & 5 \\
\hline 306 & Pselaphinae sp. 2 & DE & 1 & 0.10 & 1 & 3 & 0.13 & 3 & 4 & 0.12 & 4 \\
\hline 307 & Pselaphinae sp. 3 & DE & 5 & 0.49 & 5 & 11 & 0.48 & 6 & 16 & 0.48 & 11 \\
\hline 308 & Pselaphinae sp. 4 & DE & 4 & 0.39 & 3 & 1 & 0.04 & 1 & 5 & 0.15 & 4 \\
\hline 309 & Pselaphinae sp. 5 & $\mathrm{DE}$ & - & - & - & 1 & 0.04 & 1 & 1 & 0.03 & 1 \\
\hline 310 & Pselaphinae sp. 6 & $\mathrm{DE}$ & - & - & - & 1 & 0.04 & 1 & 1 & 0.03 & 1 \\
\hline 311 & Pselaphinae sp. 7 & $\mathrm{DE}$ & 3 & 0.29 & 3 & - & - & - & 3 & 0.09 & 3 \\
\hline 312 & Pselaphinae sp. 8 & $\mathrm{DE}$ & 1 & 0.10 & 1 & - & - & - & 1 & 0.03 & 1 \\
\hline 313 & Pselaphinae sp. 9 & $\mathrm{DE}$ & - & - & - & 1 & 0.04 & 1 & 1 & 0.03 & 1 \\
\hline 314 & Pselaphinae sp. 10 & $\mathrm{DE}$ & - & - & - & 1 & 0.04 & 1 & 1 & 0.03 & 1 \\
\hline 315 & Pselaphinae sp. 11 & DE & 1 & 0.10 & 1 & 3 & 0.13 & 3 & 4 & 0.12 & 4 \\
\hline 316 & Pselaphinae sp. 12 & $\mathrm{DE}$ & - & - & - & 3 & 0.13 & 2 & 3 & 0.09 & 2 \\
\hline 317 & Pselaphinae sp. 13 & $\mathrm{DE}$ & - & - & - & 2 & 0.09 & 2 & 2 & 0.06 & 2 \\
\hline 318 & Pselaphinae sp. 14 & $\mathrm{DE}$ & - & - & - & 2 & 0.09 & 2 & 2 & 0.06 & 2 \\
\hline 319 & Rugilus sp. 1 & DE & - & - & - & 9 & 0.39 & 5 & 9 & 0.27 & 5 \\
\hline 320 & Scaphidium flohri & MI & 2 & 0.20 & 2 & 1 & 0.04 & 1 & 3 & 0.09 & 3 \\
\hline 321 & Scaphisoma sp. 1 & MI & - & - & - & 1 & 0.04 & 1 & 1 & 0.03 & 1 \\
\hline 322 & Scaphisoma sp. 2 & MI & - & - & - & 2 & 0.09 & 2 & 2 & 0.06 & 2 \\
\hline 323 & Scopaeus sp. & $\mathrm{DE}$ & 1 & 0.10 & 1 & 4 & 0.17 & 3 & 5 & 0.15 & 4 \\
\hline 324 & Sepedophilus sp. 1 & MI & - & - & - & 7 & 0.31 & 6 & 7 & 0.21 & 6 \\
\hline 325 & Sepedophilus sp. 2 & MI & - & - & - & 2 & 0.09 & 2 & 2 & 0.06 & 2 \\
\hline 326 & Sepedophilus sp. 3 & MI & - & - & - & 1 & 0.04 & 1 & 1 & 0.03 & 1 \\
\hline 327 & Somoleptus sp. & DE & - & - & - & 1 & 0.04 & 1 & 1 & 0.03 & 1 \\
\hline 328 & Staphylinini sp. 1 & $\mathrm{DE}$ & - & - & - & 1 & 0.04 & 1 & 1 & 0.03 & 1 \\
\hline 329 & Stenus sp. 1 & $\mathrm{DE}$ & - & - & - & 29 & 1.27 & 8 & 29 & 0.88 & 8 \\
\hline 330 & Styngetus deyrollei & $\mathrm{DE}$ & 4 & 0.39 & 2 & 32 & 1.40 & 7 & 36 & 1.09 & 9 \\
\hline 331 & Tachinomorphus sp. & SA & - & - & - & 3 & 0.13 & 1 & 3 & 0.09 & 1 \\
\hline 332 & Tachynus sp. & DE/MI & - & - & - & 1 & 0.04 & 1 & 1 & 0.03 & 1 \\
\hline 333 & Thoracophorini sp. & $\mathrm{SA}$ & - & - & - & 1 & 0.04 & 1 & 1 & 0.03 & 1 \\
\hline 334 & Thoracophorus sp. & SA & - & - & - & 1 & 0.04 & 1 & 1 & 0.03 & 1 \\
\hline 335 & $\begin{array}{l}\text { Toxidium sp. } 1 \\
\text { SYDMAENIDAE }\end{array}$ & MI & - & - & - & 2 & 0.09 & 2 & 2 & 0.06 & 2 \\
\hline 336 & Sydmaenidae sp. 1 & $\mathrm{DE}$ & 16 & 1.57 & 7 & 128 & 5.59 & 17 & 144 & 4.35 & 24 \\
\hline 337 & Sydmaenidae sp. 2 & $\mathrm{DE}$ & - & - & - & 27 & 1.18 & 6 & 27 & 0.82 & 6 \\
\hline
\end{tabular}




\begin{tabular}{|c|c|c|c|c|c|c|c|c|c|c|c|}
\hline $\begin{array}{l}\text { Clave } \\
\text { - taxon }\end{array}$ & & $\begin{array}{l}\text { Hábitos } \\
\text { aliment. }\end{array}$ & & Lluvias & & & Secas & & & Total & \\
\hline 338 & $\begin{array}{l}\text { Sydmaenidae sp. } 3 \\
\text { TELEGEUSIDAE }\end{array}$ & $\mathrm{DE}$ & 1 & 0.10 & 1 & - & - & - & 1 & 0.03 & 1 \\
\hline 339 & $\begin{array}{l}\text { Pseudotelegeusis sp. } \\
\text { TENEBRIONIDAE }\end{array}$ & SA & - & - & - & 4 & 0.17 & 4 & 4 & 0.12 & 4 \\
\hline 340 & Alleculini sp. & $\mathrm{OM}$ & - & - & - & 2 & 0.09 & 2 & 2 & 0.06 & 2 \\
\hline 341 & Statira sp. & OM & - & - & - & 1 & 0.04 & 1 & 1 & 0.03 & 1 \\
\hline 342 & Lagriini sp. & $\mathrm{OM}$ & - & - & - & 1 & 0.04 & 1 & 1 & 0.03 & 1 \\
\hline 343 & Tenebrionidae sp. 1 & $\mathrm{OM}$ & 5 & 0.49 & 4 & 2 & 0.09 & 2 & 7 & 0.21 & 6 \\
\hline 344 & Tenebrionidae sp. 2 & $\mathrm{OM}$ & 1 & 0.10 & 1 & - & - & - & 1 & 0.03 & 1 \\
\hline 345 & $\begin{array}{l}\text { Tenebrionidae sp. } 3 \\
\text { THROSCIDAE }\end{array}$ & $\mathrm{OM}$ & - & - & - & 1 & 0.04 & 1 & 1 & 0.03 & 1 \\
\hline 346 & Trixagus sp. & DE & - & - & - & 3 & 0.13 & 2 & 3 & 0.09 & 2 \\
\hline 347 & Throscidae sp. 1 & $\mathrm{DE}$ & - & - & - & 1 & 0.04 & 1 & 1 & 0.03 & 1 \\
\hline 348 & Throscidae sp. 2 & $\mathrm{DE}$ & - & - & - & 1 & 0.04 & 1 & 1 & 0.03 & 1 \\
\hline 349 & Throscidae sp. 3 & $\mathrm{DE}$ & - & - & - & 2 & 0.09 & 1 & 2 & 0.06 & 1 \\
\hline 350 & $\begin{array}{l}\text { Throscidae sp. } 4 \\
\text { TROGOSITIDAE }\end{array}$ & $\mathrm{DE}$ & - & - & - & 1 & 0.04 & 1 & 1 & 0.03 & 1 \\
\hline 351 & $\begin{array}{l}\text { Trogositidae sp. } \\
\text { ZOPHERIDAE }\end{array}$ & DE & - & - & - & 1 & 0.04 & 1 & 1 & 0.03 & 1 \\
\hline 352 & Monommatini sp. & SA/MI & - & - & - & 1 & 0.04 & 1 & 1 & 0.03 & 1 \\
\hline
\end{tabular}

especie dominante, cuya abundancia representó el $13.95 \%$ de la abundancia total. Otras especies importantes fueron Aleocharinae sp. 8 (Staphylinidae), Ontherus mexicanus (Scarabaeidae), Sphaeridiinae sp. 1, (Hydrophilidae) Deltochilum mexicanum (Scarabaeidae) y Nicrophorus olidus (Silphidae). El ensamblaje de lluvias contiene también un alto número de especies raras $(88.05 \%)$.

Durante la época de secas, la estructura de la dominancia mostró a Aleocharinae sp. 8 (Staphylinidae) como la especie dominante, cuya abundancia representó el $13.41 \%$ de la abundancia total. Otras especies importantes fueron Scydmaenidae sp. 1 (Scydmaenidae), Philonthus sp. 1 (Staphylinidae) y Nitidula sp. 1 (Nitidulidae). El ensamblaje de la temporada seca contiene un elevado número de especies raras (91.94\%).

En relación con la abundancia, se recolectaron 1018 (30\%) coleópteros de la época de lluvias y 2290 (70\%) de la época de sequía. La familia con mayor abundancia fue Staphylinidae con 1521 ejemplares, seguida de Nitidulidae con 366 y Scarabaeidae con 220.

El ANOVA no paramétrico o prueba de KruskalWallis mostró efecto significativo del factor temporada sobre la abundancia $\left(C h i^{2}=12.13, \mathrm{gl}=1, p<0.01\right)$, siendo el número promedio de coleópteros para la temporada de lluvias de 50.9 individuos/trampa y para la de sequía, de 114.5 individuos/trampa. De la misma forma, la prueba de Kruskal Wallis evidenció efecto significativo del factor mes sobre la abundancia de coleópteros $\left(C h i^{2}=22.35\right.$, $\mathrm{gl}=7, p<0.01)$. Los contrastes pareados mediante la prueba U de Mann-Whitney mostraron que la mayoría de las diferencias radicaron entre los meses de marzo, abril y mayo con los restantes meses (Fig. 6).

La similitud entre los ensamblajes de secas y lluvias fue de $34.40 \%$. (Bray-Curtis). Los mayores valores de similitud (y por ende las menores tasas de recambio) se encuentran entre los meses contiguos de junio, julio, agosto y septiembre que corresponden a la temporada de lluvias (Cuadro 2), y el menor grado de similitud (mayor tasa de recambio) entre los meses contiguos de febrero, marzo, abril y mayo que, a su vez, corresponden a la temporada de secas. Al parecer, los meses que conformaron la época de secas (2005) representan una fauna característica, mientras que aquellos de lluvias (2006) conforman otra fauna.

La estimación de la riqueza gamma mediante el modelo de dependencia lineal estimó 364 taxa (12 más), mientras que el modelo de Clench estimó 494 (142 más), lo cual arroja una eficiencia del muestreo de 96.44 y $71.26 \%$, respectivamente (Fig. 7). De acuerdo con los coeficientes de determinación $R^{2}$ (Cuadro 3), el modelo de dependencia lineal explicó mejor el comportamiento de los datos. Las pendientes para ambas curvas fueron 1.67 (dependencia lineal) y 2.60 (Clench).

El análisis de correspondencias evidenció asociación de morfoespecies y meses (inercia total $=0.55, \mathrm{Chi}^{2}{ }_{280}=$ $332.8, p=0.02$ ). Las 2 primeras dimensiones resultaron significativas y explicaron en conjunto el $58.49 \%$ de la variación de los datos (Fig. 8). La primera dimensión separó (lado derecho del origen) los meses de junio, julio, agosto y septiembre que conforman la temporada de lluvias. Diversos taxa (véase clave numérica en el Cuadro 2) 


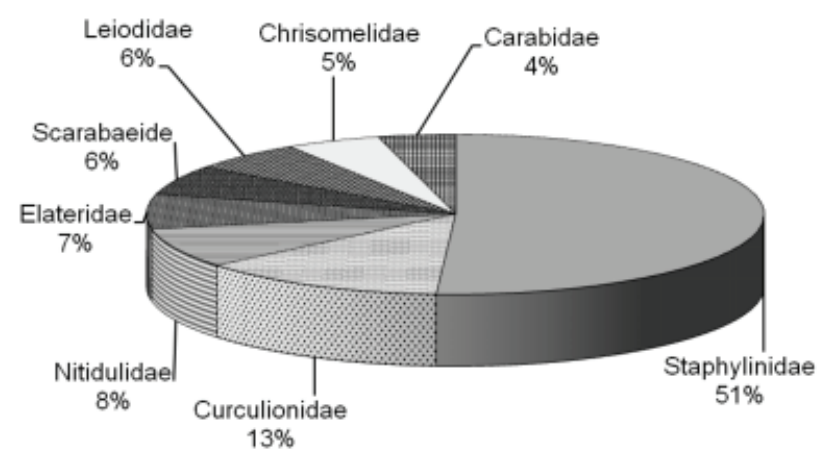

Figura 2. Familias con más de diez morfoespecies recolectadas.

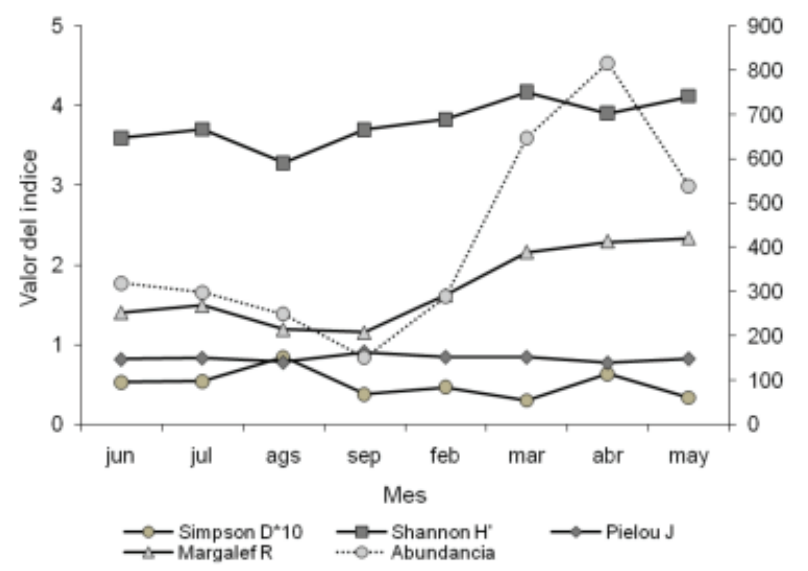

Figura 4. Variación de los índices de diversidad entre los meses de recolecta. La dominancia $D$ está multiplicada por 10 y la riqueza dividida por 10 para hacer estos índices comparables en la gráfica, la abundancia está dividida entre 100.

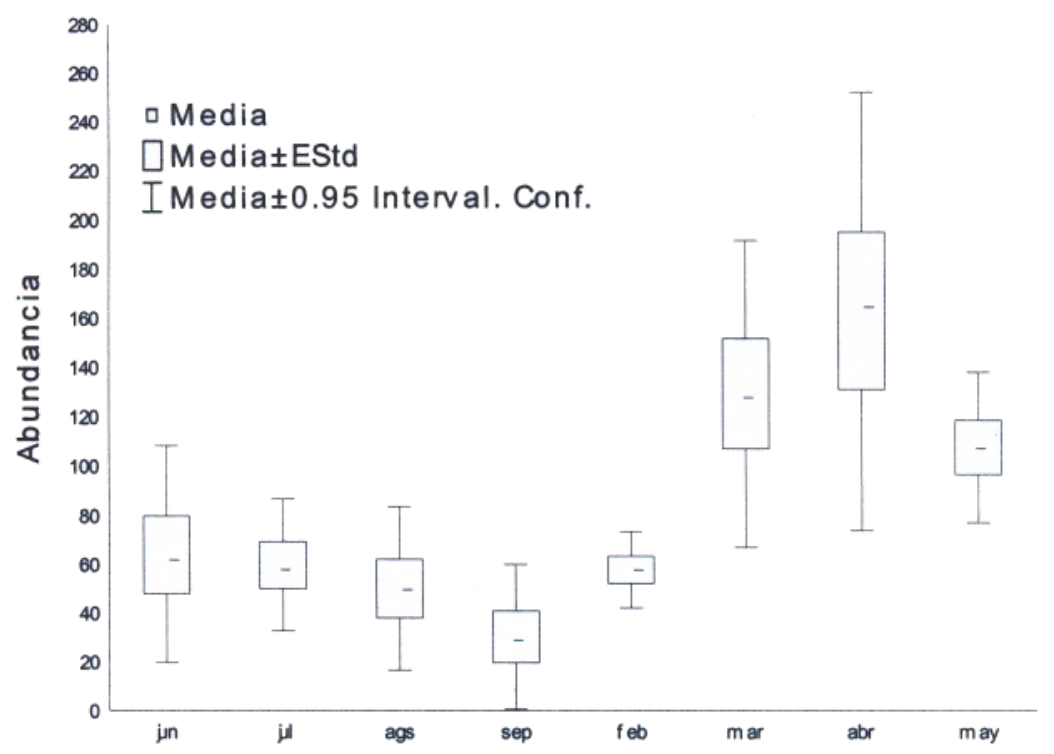

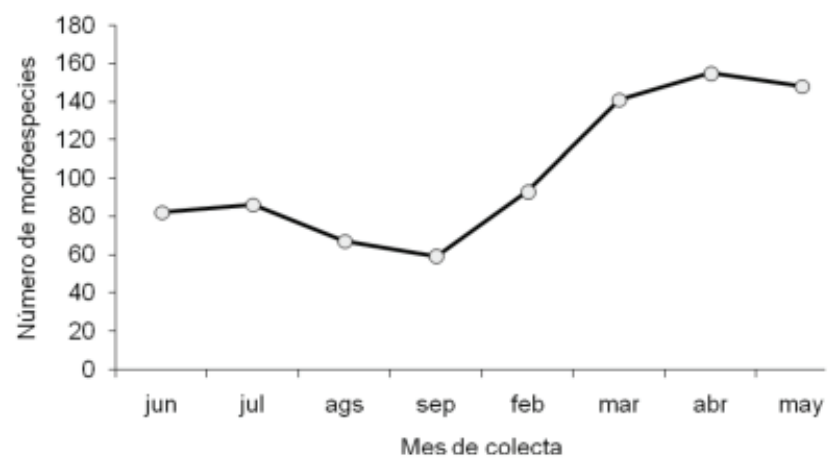

Figura 3. Variación del número de morfoespecies de Coleoptera de Tlanchinol, Hidalgo, recolectadas durante la época de lluvias y de sequía.

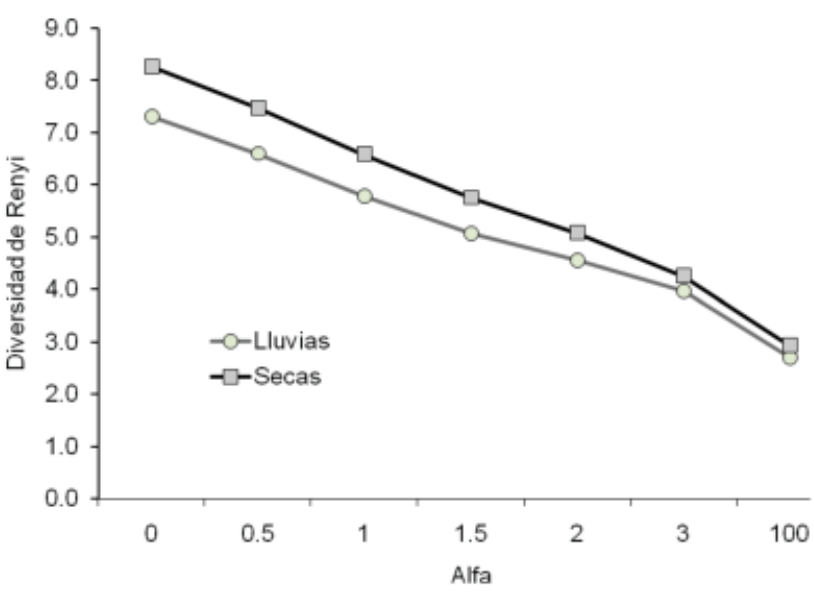

Figura 5. Perfiles de diversidad estacional de Renyi para los ensamblajes de coleópteros de Tlanchinol, Hidalgo.
Figura 6. Medias e intervalos de confianza al 95\% para la abundancia mensual de coleópteros capturados con trampas de intercepción de vuelo en Tlanchinol, Hidalgo durante 2005-2006. 
Cuadro 3. Parámetros generados por los modelos de Clench y de dependencia lineal para los ensamblajes total y por temporada de Coleoptera de Tlanchinol, Hidalgo, México, capturados con trampas de intercepción de vuelo (TIV)

\begin{tabular}{|c|c|c|c|c|c|c|c|c|c|c|c|c|c|c|}
\hline & \multirow[t]{2}{*}{$S_{o b s}$} & \multicolumn{3}{|c|}{ Clench } & \multicolumn{10}{|c|}{ Dependencia lineal } \\
\hline & & $n$ & $a$ & $b$ & $a / b$ & $\mathrm{R}^{2}$ & ef & $\mathrm{pd}$ & $a$ & $b$ & $a / b$ & $\mathrm{R}^{2}$ & ef & $\mathrm{Pd}$ \\
\hline Total & 352 & 40 & 28.67 & 0.058 & 494 & 78.22 & 71.26 & 2.60 & 24.37 & 0.067 & 364 & 98.91 & 96.44 & 1.71 \\
\hline Lluvias & 159 & 20 & 21.1 & 0.08 & 253 & 99.9 & 62.9 & 2.98 & 18.8 & 0.11 & 177 & 99.8 & 89.8 & 2.26 \\
\hline Secas & 310 & 20 & 43.2 & 0.09 & 474 & 99.9 & 65.40 & 5.43 & 38.1 & 0.11 & 337 & 99.8 & 92 & 3.94 \\
\hline
\end{tabular}

$n=$ esfuerzo total de muestreo; $S_{o b s}=$ número de morfoespecies observadas, $a=$ la pendiente al inicio del muestreo; $b=$ un parámetro relacionado con la manera en la que se acumulan las nuevas especies durante el muestreo; $a / b=$ asíntota; $R^{2}=$ coeficiente de determinación; ef = porcentaje de eficiencia y $p d=$ pendiente. La pendiente fue evaluada utilizando las primeras derivadas de las funciones de Clench $\left[\mathrm{a} /(1+\mathrm{b} * \mathrm{n})^{2}\right]$, de dependencia lineal $[a * \exp (-b * n)]$.

tienden a asociarse con estos meses. De la misma forma, el lado izquierdo contiene los meses de febrero, marzo, abril y mayo que conforman la época de secas.

Algunos taxa mostraron una clara afinidad por la temporada de lluvias como lo fueron Thalycra sp. 1, Sphaeridiinae sp. 1, Ontherus mexicanus, Deltochilum mexicanum, Aphodinae sp. 1 y Dichotomius satanas; mientras que otros lo hicieron por la temporada de sequía, como Scydmaenidae sp. 1, Ecarinoespherula sp. 1, Anotylus sp. 1, Aleocharinae sp. 20, Carabidae sp. 3, Philonthus sp. 3, Heterothops sp., Neohypnus sp., Bryophorus sp., Nitidulidae sp. 4, Aleocharinae sp. 3, Lordithon nubicola, entre otras.

En cuanto a la abundancia de los grupos funcionales se observó que el $54 \%$ de los organismos recolectados se ubicaron en la categoría de los depredadores con 132 morfoespecies (Figs. 9 y 10), el resto de la abundancia varió entre 3\% (saprófagos) y 12\% (saprómicófagos), mientras que en número de especies siguieron fitófagos (59) y micófagos (52).

\section{Discusión}

El ensamblaje de coleópteros que aquí se presenta para el bosque mesófilo de Tlanchinol representa uno de los más grandes registrados hasta ahora en cuanto a riqueza y composición. La estructura de la dominancia es característica de un ensamblaje que proviene de una localidad con excelente calidad ecológica, pocas especies dominantes, un grupo pequeño de especies codominantes y un gran número de especies raras; a diferencia de los sitios con disturbio producido por actividad humana, cuyos ensamblajes se caracterizan por tener una o algunas especies claramente dominantes, un grupo pequeño de especies codominantes y pocas especies raras. Algunos taxa suelen tener gran éxito dentro de las nuevas condiciones de disturbio y ocupan un claro lugar en la dominancia, con una o 2 especies representando más de la mitad de la abundancia total.

En general se sabe que el bosque mesófilo es rico en especies tanto vegetales como animales, siendo este trabajo un claro ejemplo de ello. Desafortunadamente, la superficie que este tipo de asociación vegetal representa en nuestro país es cada vez más reducida. Se sabe también que no es fortuito que un grupo como Coleoptera contenga una de las mayores riquezas de especies en prácticamente cualquier tipo de ambiente. La mayor parte de la biodiversidad animal de un área puede caracterizarse atendiendo al estudio de grupos como Coleoptera, Lepidoptera, Diptera e Hymenoptera (May, 1988; Erwin, 1991). Tal vez estos grupos en conjunto representen hasta un $80 \%$ de la fauna de cualquier área.

El alto número de especies recolectadas y la estructura del ensamblaje característico de un ecosistema conservado, no sólo se deben a la gran diversidad de los bosques mesófilos sino a la gran eficiencia de recolección de las TIV para los coleópteros, la cual ya ha sido documentada (Juillet, 1963; Hosking, 1979; Basset, 1988; Basset y Arthington, 1992; Hill y Cermak, 1997; McWilliam y Death, 1998; Leksono et al., 2005), pero no para estudios hechos en México. Esperamos que esta metodología pueda ser utilizada con mayor frecuencia en estudios futuros, que permitan hacer comparaciones y abordar distintas variables, como su ubicación en un rango altitudinal, en distintos tipos de ecosistemas, bajo distintos grados de conservación, etcétera.

Particularmente, el hecho de que Staphylinidae presentara el mayor número de morfoespecies quizá se debe a que es una de las familias de coleópteros más ricas en especies, ya que tiene más de 42000 descritas en el mundo, de las cuales 1522 se han citado para México (Klimaszewski, 1996; Navarrete-Heredia y ZaragozaCaballero, 2006). También biológicamente es muy 

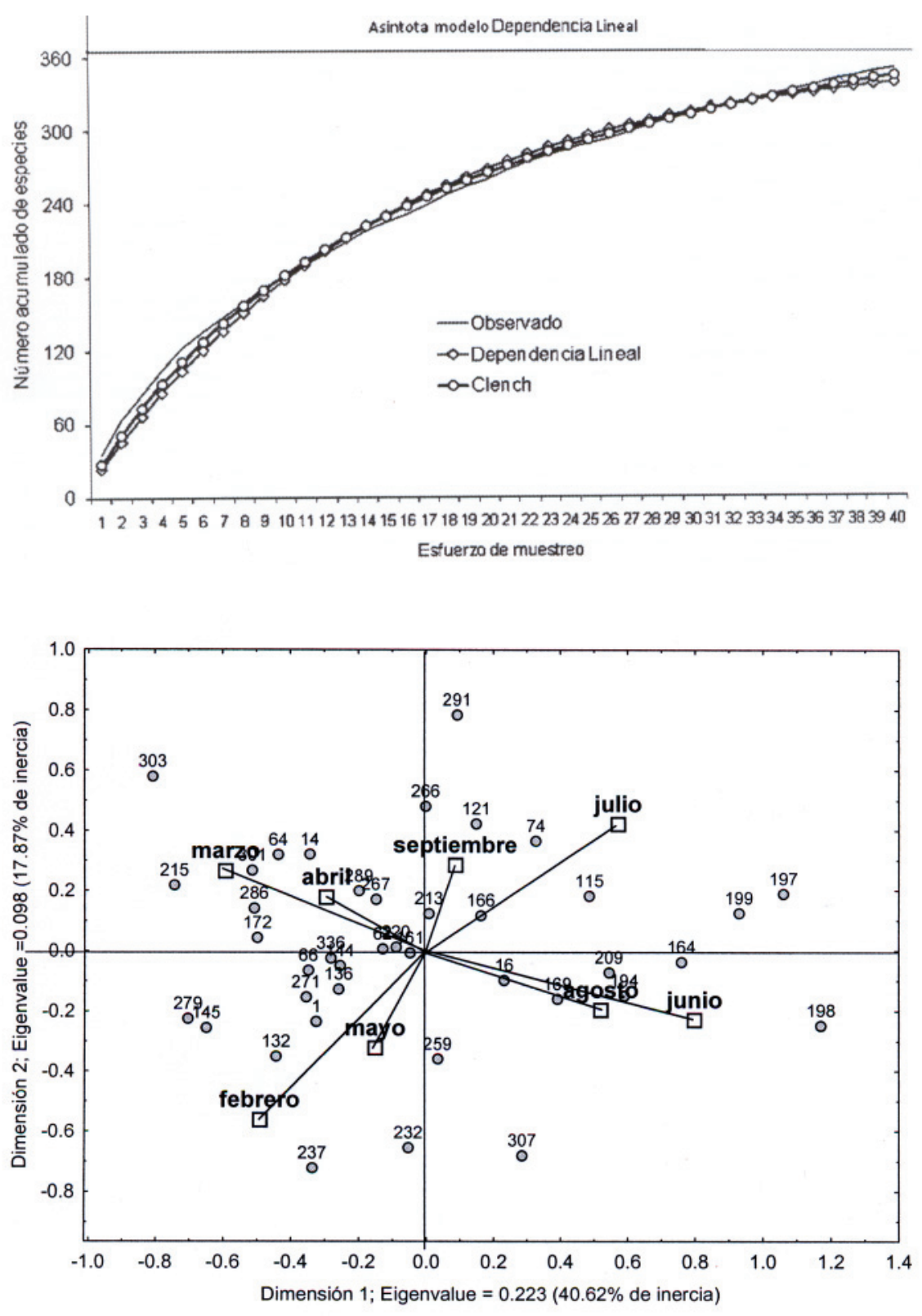

Figura 7. Curvas de acumulación de morfoespecies generadas por los modelos de dependencia lineal (rombos) y Clench (círculos) para el ensamblaje total de coleópteros capturados con trampas de intercepción de vuelo (TIV) en Tlanchinol, Hidalgo, México.

Figura 8. Análisis simple de correspondencias que muestra las asociaciones de los taxa de Coleoptera de Tlanchinol con los meses de recolecta. Clave de las morfoespecies en el Cuadro 2.

puede encontrar en cualquier tipo de hábitat: bajo la corteza de los árboles, flores, carroña, en la hojarasca del bosque, en hongos y frutos en descomposición, además de que sus hábitos alimentarios pueden ser micófagos o saprófagos (Habeck, 2002; Solís, 2002).

El que la mayor riqueza de morfoespecies ocurriera en la época de sequía y no en la de lluvias puede atribuirse al mayor número de taxa de talla pequeña (menores a $5 \mathrm{~mm}$ ) durante la primera estación en comparación con la segunda

diversa, se le encuentra en diferentes tipos de hábitats y sus representantes pueden alimentarse de una amplia gama de recursos (White, 1983; Solís, 2002). La segunda familia con mayor riqueza de morfoespecies fue Curculionidae; probablemente se deba a que es la familia con el mayor número de especies de todo Coleoptera, y a que se le puede encontrar en diferentes tipos de hábitat incluyendo el acuático; tiene representantes de hábitos diurnos y nocturnos, y la mayoría de sus especies son fitófagas, alimentándose de cualquier parte viva o muerta de las plantas (Solís, 2002). El tercer lugar en riqueza de morfoespecies fue Nitidulidae, tal vez porque es una familia que se le (lluvias), posiblemente porque es cuando logran desplazarse con mayor facilidad para buscar sus distintos recursos, ya que un evento de lluvia puede impedir el vuelo de estos insectos pequeños; así como al hecho de que estos bosques mantienen considerable humedad ambiental durante todo el año gracias a su neblina característica. Aunque no debe descartarse la posibilidad de que los coleópteros de tallas grandes puedan caer fuera del recipiente colector o incluso salir del líquido colector, esta probabilidad sería la misma para ambas temporadas de recolección y no existen datos en la literatura al respecto, por lo que sería pertinente hacer observaciones de campo. 


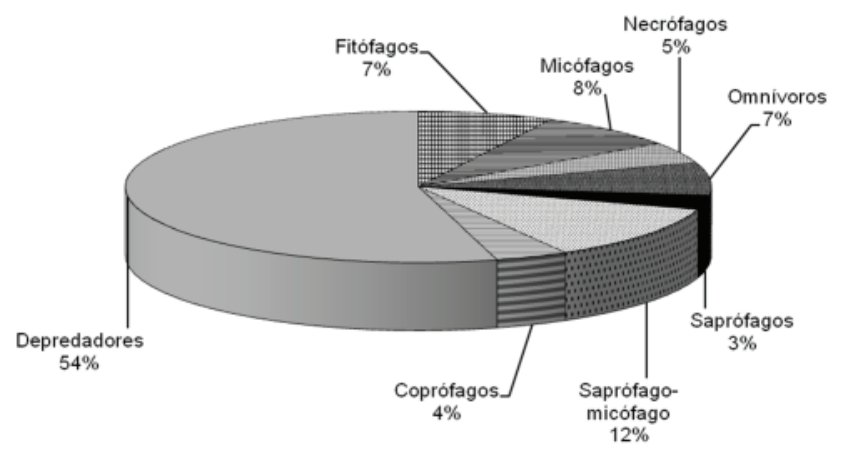

Figura 9. Reparto de la abundancia en los grupos tróficos de Coleoptera de Tlanchinol, Hidalgo.

Al comparar el número de morfoespecies recolectadas en cada mes, se aprecia que el valor máximo fue en abril. Se sabe que en los bosques mesófilos de montaña los periodos de lluvia y sequía no están bien delimitados (Luna et al., 2001). En Tlanchinol, las primeras lluvias del año 2005 ocurrieron en abril y no fueron tan intensas como las de los meses posteriores a junio. Quizá ese factor influyó para que en dicho mes se recolectara un mayor número de morfoespecies, ya que probablemente estas lluvias ayudaron a la aparición de nuevos recursos vegetales (frutos, flores, brotes, etc.), así como al surgimiento de cuerpos fructíferos de algunas especies de hongos.

Algunos trabajos que comparan la fauna durante las temporadas del año (por ejemplo Márquez, 1994, 1998; Pérez, 1996) han encontrado una mayor riqueza y abundancia de organismos en la época de lluvias y argumentan que se debe a que es la época con mayor cantidad de recursos alimenticios disponibles. En este trabajo los resultados son totalmente diferentes a los anteriores, ya que de manera significativa, los valores de riqueza de morfoespecies, abundancia y diversidad fueron más favorables para la época de sequía que para la de lluvias, posiblemente debido a que en el bosque mesófilo de montaña los periodos secos son cortos y poco intensos. Aunque se reduce la humedad del suelo y existe poca precipitación, la nubosidad en forma de neblina reduce la evaporación, ayudando a que exista agua durante periodos de poca lluvia, lo que influye para que en estos bosques nunca exista deficiencia marcada de humedad (Luna et al., 2001). Posiblemente debido a lo anterior, los organismos que ahí habitan nunca tienen una fuerte escasez de alimento.

Si se compara la distribución de los organismos en las 2 épocas de estudio con base en su tamaño, se nota que los organismos pequeños (longitud menor a $5 \mathrm{~mm}$ ) son más abundantes y la mayoría se recolectaron en la época

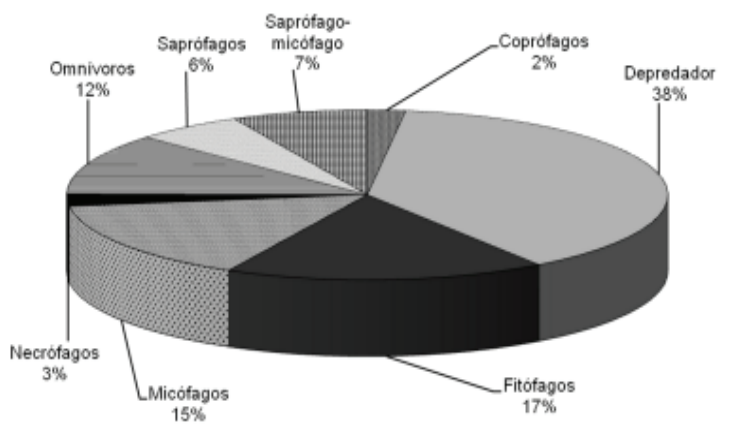

Figura 10. Número de morfoespecies por grupo trófico de Coleoptera de Tlanchinol, Hidalgo.

de sequía, mientras que los grandes (longitud mayor a 10 $\mathrm{mm}$ ) en su mayoría fueron recolectados en la época de lluvias, lo que hace suponer que los organismos pequeños difícilmente se pueden desplazar en la época de lluvias, porque las gotas de agua llegan a superar incluso su peso corporal. Un ejemplo que sustenta esta hipótesis es el caso de la familia Ptiilidae que incluye organismos cuya talla es la más pequeña de todo Coleoptera; se caracterizan por poseer alas membranosas en forma de pluma (White, 1983), que al abrirlas permiten su dispersión a hábitats más favorables (Hall, 2001). Esto indica que sólo pueden desplazarse cuando las condiciones climáticas son favorables para ellos (sin lluvia). En este trabajo se recolectaron 21 ejemplares de esta familia y todos ellos fueron capturados en la época de sequía.

Las trampas fueron ubicadas en la parte más conservada del bosque; si se considera que la zona de estudio se encuentra rodeada por un área deforestada. Es posible que los individuos en la época de sequía se hayan refugiado en esta parte del bosque, evitando condiciones de altas temperaturas y escasez de alimento que existen alrededor del bosque.

En este trabajo, la mayor abundancia de organismos y la mayor riqueza de morfoespecies coincidieron en la época de sequía. Cabe destacar que no siempre la época o lugar con mayor riqueza específica tiene la mayor abundancia (Márquez, 1998). En congruencia con la riqueza de especies, el hecho de que la abundancia haya sido mayor en la época de sequía que en la de lluvias puede deberse a que las lluvias escasas o nulas fueron un factor determinante que propició el desplazamiento de los organismos más pequeños, mientras que durante la estación lluviosa, la mayor precipitación afectó negativamente estos desplazamientos. Además, se notó que las familias más ricas en especies de coleópteros, tienen en su mayoría representantes pequeños, como es el caso de Staphylinidae 
y Nitidulidae, quienes fueron las que presentaron mayor abundancia de individuos.

Algunas especies mostraron estacionalidad marcada, apareciendo sólo en una de las 2 estaciones del año, o presentando poblaciones más altas en una época con respecto a otra. De las 20 morfoespecies más abundantes en cada época, sólo se comparten siete. La estacionalidad es un factor que podría ayudar a reducir la competencia por los recursos alimenticios. Por otro lado, la similitud de la fauna entre estaciones se puede atribuir a que el área de estudio se encuentra cerca de la línea ecuatorial y según Wolda (1988), en las zonas tropicales es muy común el multivoltismo, que consiste en tener varias generaciones por año; así como el traslape de generaciones y que la mayoría de las especies se mantengan activas durante la mayor parte del año.

La lista de coleópteros aquí registrada se incrementaría si se anexaran coleópteros con distintos hábitos de vuelo o que no vuelan (formas ápteras, braquípteras, acuáticas o que vuelan a niveles superiores de donde se instalaron las trampas); sin embargo, es muy complicado estudiar todos los coleópteros de cualquier localidad. Resulta notable que a partir de una superficie relativamente pequeña de muestreo $\left(1.43 \mathrm{~m}^{2}\right.$ por 5 trampas funcionando durante 5 días) se lograra registrar un ensamblaje tan grande. Si se considera que para el país se han registrado 114 familias de coleópteros (Navarrete-Heredia y Fierros-López, 2001), este trabajo estaría registrando el $43.8 \%$ del total de familias de coleópteros reconocidas para el país.

A pesar de que no se alcanzó la asíntota con los estimadores utilizados, se considera que el esfuerzo de muestreo logró listar un elevado número de taxa de Coleoptera. Sin embargo, si se aumentara el número de trampas en el bosque y se colocaran a diferentes alturas, probablemente se mejorarían los resultados, acercándose cada vez más a los resultados que predicen los estimadores utilizados.

Inicialmente, con base en el coeficiente de determinación, hemos elegido el modelo que mejor ajustó nuestros datos; seguidamente, y para evaluar la completitud de nuestro listado, hemos atendido a la pendiente de la curva evaluada en el máximo esfuerzo realizado $(n=40)$ utilizando para ello la primera derivada de cada función. Este modelo fue el de dependencia lineal $\left(\mathrm{R}^{2}=98.91\right)$, el cual también tuvo la menor pendiente (Cuadro 3). Cuando evaluamos las pendientes para los ensamblajes de las temporadas se observa que el ensamblaje de lluvias está más completo que el de secas. La velocidad a la cual los taxa se estaban adicionando a la lista durante el muestreo en la temporada de secas indica que aún en la vigésima muestra un elevado número de taxa fue registrado. Estas pendientes son en general grandes (Cuadro 3) si se considera que un listado casi completo tiene una pendiente inferior a 0.1 , pero esto puede ser también un efecto del tamaño de la muestra utilizado. Nuestra matriz de datos tiene pocas columnas (muestras) y muchos renglones (taxa), y realmente no existe un grupo de muestras a partir de las cuales la adición de taxa disminuyera notablemente. Los taxa se acumularon a grandes saltos consecutivos a la lista. Creemos que esto es bueno desde el punto de vista faunístico, pero tal vez este tipo de grandes muestras no producen un efecto asintótico evidente a pesar de que la lista puede estar bastante completa. Generalmente el efecto asintótico se aprecia mejor con aquellas matrices que tienen muchas columnas (muestras) y un menor número de renglones (taxa). Son muestras más pequeñas en donde la acumulación de taxa es gradual y progresiva existiendo, después de cierto esfuerzo, una disminución gradual de nuevos taxa.

Los coleópteros han logrado conquistar distintos ambientes debido principalmente a que se pueden alimentar de una gran variedad de alimentos (Borror y Dwight, 1971; Márquez, 2004). Además, patrones de abundancia de grupos funcionales como el que se observó en nuestro estudio, también han sido registrados en otros trabajos con insectos como el de Pérez (1996) y Martínez (2006). El hecho de que los depredadores hayan sido la categoría en que se ubicaron la mayoría de las morfoespecies quizá se debe a que difícilmente llegan a carecer de alimento, pues todo el tiempo existen comunidades de artrópodos con los cuales alimentarse. Aunque la densidad de poblaciones llegue a fluctuar debido a la escasez de alimento, nunca desaparecen por completo porque mientras algunas poblaciones son abundantes en una época, otras son abundantes en una época distinta. Asimismo, se sugiere que los depredadores vuelan con mayor frecuencia que los coleópteros de otros hábitos alimenticios, a la altura del bosque a la que se instalaron las trampas; muestra de ello es la familia Staphylinidae, quien tuvo la mayor riqueza y abundancia de morfoespecies, la mayoría de sus representantes recolectados presentan este hábito alimenticio; además de que los depredadores necesitan desplazarse más en busca de alimento, que los coleópteros ubicados en otras categorías.

Las categorías "fitófago" y "micófago" fueron de las mejor representadas quizá porque las plantas y los hongos son de los recursos más abundantes en el bosque mesófilo de montaña, debido a la humedad que presenta la mayor parte del año. Existen pocas publicaciones relacionadas con este tema, por lo que no es fácil comparar los resultados de este trabajo con otros, y las diferencias entre ellos pueden deberse a que los objetivos de cada investigación son diferentes. Sin embargo, con base en comparaciones con otros trabajos (Cuadro 4), se considera que el muestreo 
Cuadro 4. Comparación de los resultados obtenidos en el presente trabajo con los de otros autores

\begin{tabular}{|c|c|c|c|c|}
\hline Autores y área de estudio & Grupos analizados & Núm. familias & Núm. especies & Núm. ejemplares \\
\hline Pedraza et al., Tlanchinol, Hidalgo & Coleoptera & 50 & 352 & 3,308 \\
\hline $\begin{array}{l}\text { Hosking (1979): Nueva Zelanda, Parque } \\
\text { Forestal Estatal Whakarewarewa }\end{array}$ & Coleoptera & 13 & 29 & 837 \\
\hline Márquez (1994): Morelos, México. & $\begin{array}{l}\text { Coleoptera en detritos de } \\
\text { Atta mexicana }\end{array}$ & 17 & 95 & 5,658 \\
\hline Pérez (1996): Chamela, Jalisco. & Coleoptera & 60 & No reportado & 8,711 \\
\hline $\begin{array}{l}\text { Chatzimanolis et al. (2004): Panamá, } \\
\text { Isla de Barro Colorado. }\end{array}$ & Staphylinidae & 1 & 35 & 1,349 \\
\hline $\begin{array}{l}\text { Canizal Ordáz (2008): Tlanchinol, } \\
\text { Hidalgo }\end{array}$ & Coleoptera & 42 & 186 & 3,213 \\
\hline
\end{tabular}

realizado en el bosque mésofilo de montaña de Tlanchinol, utilizando TIV, aportó una alta diversidad de organismos en comparación con el número de coleópteros recolectados en otros trabajos y hacen de este sitio un lugar biológicamente importante y prioritario para su conservación.

El diseño de una red de reservas debería basarse en múltiples criterios (Moreno y Halffter, 2000). La información debe proceder de la mayor variedad de organismos posibles, incluyendo principalmente a los insectos, grupo taxonómico representativo de la biodiversidad terrestre, y es también necesario incluir distintos atributos de la diversidad biológica (número de especies, rareza, endemicidad, diversidad filogenética, especies en peligro de extinción, entre otros). Si se considera al ensamblaje de Coleoptera aquí reportado como indicador de diversidad, sugerimos que el bosque mesófilo de Tlanchinol se encuentra en un buen estado de conservación que debería ser protegido y estudiado.

\section{Agradecimientos}

Agradecemos a R. Vergara y a C. Ortiz por su apoyo en el trabajo de campo; a J. Asiain (Centro de Investigaciones Biológicas, UAEH) por la revisión crítica del manuscrito y la identificación de algunos coleópteros; a J. Bueno (Centro de Investigaciones Biológicas, UAEH), por la revisión del resumen y abstract. A los siguientes especialistas, por su ayuda en la identificación de coleópteros: S. Zaragoza (Instituto de Biología, UNAM, Cantharidae, Lampyridae, Phengodidae y Telegeusidae); L. Delgado y R. Arce (Instituto de Ecología, A. C., Scarabaeidae y Dytiscidae, respectivamente) y L. Herman (Museo Americano de Historia Natural, Staphylinidae). A dos revisores anónimos, por los comentarios valiosos aportados para mejorar nuestro trabajo. Juan Márquez agradece el apoyo económico otorgado por el CONACYT a través del proyecto "Sistemática y biogeografía de Staphylinidae (Insecta: Coleoptera) del estado de Hidalgo", clave 90056.

\section{Literatura citada}

Arnett, R. H. y M. C. Thomas. (eds.). 2001. American beetles. Archostemata, Myxophaga, Adephaga, Polyphaga: Staphyliniformia, vol. 1. CRC, Boca Raton, Florida. 443 p.

Arnett, R. H., M. C. Thomas, P. Skelley y J. H. Frank. (eds.). 2002. American beetles. Polyphaga: Scarabaeoidea through Curculionoidea, vol. 2. CRC, Boca Raton, Florida. 861 p.

Asiain, J. y J. Márquez. 2003. Revisión sistemática y análisis filogenético del género Misantlius Sharp, 1885 (Coleoptera: Staphylinidae: Staphylinini). Folia Entomológica Mexicana 42: 37-64.

Balselga, A. y F. Novoa. 2008. Coleoptera in a relict forest of Spain: implications of hyperdiverse taxa for conservation strategies. Annals of the Entomological Society of America 101: 402-410.

Basset, Y. 1988. A composite interception trap for sampling arthropods in tree canopies. Journal of the Entomological Society of Australia 27: 213-219.

Basset, Y. y A. H. Arthington. 1992. The arthropod community of an Australian rainforest tree: abundance of component taxa, species richness and guild structure. Australian Journal of Ecology 17: 89-98.

Borror, D. J. y D. Dwight. 1971. An introduction to the study of insects. Holt, Rinehart and Winston, Austin, Texas. 864 p.

Canizal Ordáz, G. A. 2008. Fauna de coleópteros (Insecta: Coleoptera) asociados al dosel del bosque mesófilo de montaña en Tlanchinol, Hidalgo, México. Tesis, Instituto de Ciencias Básicas e Ingeniería, Universidad Autónoma del Estado de Hidalgo, Pachuca. 103 p.

Challenger, A. 1998. Utilización y conservación de los ecosistemas terrestres de México. Comisión Nacional para el Conocimiento y Uso de la Biodiversidad/ Instituto de Biología, UNAM/Agrupación Sierra Madre, México, D.F. $847 \mathrm{p}$.

Chatzimanolis, S., J. S. Ashe y R. Hanley. 2004. Diurnal/nocturnal 
activity of rove beetles (Coleoptera: Staphylinidae) on Barro Colorado Island, Panama assayed by flight intercept trap. The Coleopterists Bulletin 58:569-577.

Colwell, R. K. 2005. EstimateS, Version 7.5: statistical estimation of species richness and shared species from samples (Software and user's guide). Freeware published at http://viceroy.eeb. uconn.edu/estimates; última consulta 14.0I.2010.

de Carvalho, M. R., F. A. Bockmann, D. S. Amorim, M. de Vivo, M. de Toledo-Piza, N. A. Menezes, J. L. de Figueiredo, R. M. C. Castro, A. C. Gill, J. D. McEachran, et al. 2005. Revisiting the taxonomic impediment. Science (Washington, D.C.) 307:353-353.

Delgado, L. y J. Márquez. 2006. Estado del conocimiento y conservación de los coleópteros Scarabaeoidea (Insecta) del Estado de Hidalgo, México. Acta Zoológica Mexicana (n.s.) 22:57-108.

Erwin, T. L. 1991. How many species are there-revisited. Conservation Biology 5:330-333.

Escobar, S. F. 2000. Diversidad de coleópteros coprófagos (Scarabaeidae: Scarabaeinae) en un mosaico de hábitats en la Reserva Natural Nukak Guaviare, Colombia. Acta Zoológica Mexicana (nueva serie) 79:103-121.

Fierros-López, H. E. 2005. Revisión del género Scaphidium Oliver, 1790 (Coleoptera: Staphylinidae) de México y Centroamérica. Dugesiana 12:1-152.

González, J. y A. Basélga. 2007. Diversity of water beetles (Coleoptera: Gyrinidae, Haliplidae, Noteridae, Hygrobiidae, Dytiscidae, and Hydrophilidae) in Galicia, Northwest Spain: estimating the completeness of the Regional Inventory. The Coleopterists Bulletin 61:95-110.

Habeck, D. 2002. Nitidulidae Latreille 1802. In American beetles. Polyphaga: Scarabaeoidea through Curculionoidea, vol. 2, R. H. Arnett, M. C. Thomas, P. E. Skelley y J. H. Frank. (eds.), CRC, Boca Raton, Florida. p. 311-315.

Hall, E. 2001. Ptiliidae Erichson, 185. In American beetles. Archostemata, Myxophaga, Adephaga, Polyphaga: Staphyliniformia, vol. 1, R. H. Arnett, M. C. Thomas, P. E. Skelley y J. H. Frank (eds.). CRC, Boca Raton, Florida. p. 233-246.

Hill, C. J. y M. Cermak. 1997. A new design and some preliminary results for a flight intercept trap to sample forest canopy arthropods. Australian Journal of Entomology 36:51-55.

Hortal, J. y J. M. Lobo. 2005. An ED-based protocol for optimal sampling of biodiversity. Biodiversidad y Conservación, 14:2913-2947.

Hosking, G. P. 1979. Trap comparison in the capture of flying Coleoptera. New Zeland Entomologist 7:87-92.

Jiménez-Valverde, A. y J. Hortal. 2003. Las curvas de acumulación de especies y la necesidad de evaluar la calidad de los inventarios biológicos. Revista Ibérica de Aracnología 8:151-161.

Juillet, J. A. 1963. A comparison of four types of traps used for capturing flying insects. Canadian Journal of Zoology 41:219-223.

Klimaszewski, J. 1996. A review of the New Zealand rove beetles (Coleoptera: Staphylinidae). New Zealand Journal of Zoology 23: 143-160.

Lawrence, J. F. y A. F. Newton. 1995. Families and subfamilies of Coleoptera (with selected genera, notes, references and data on family-group names). In Biology, phylogeny and classification of Coleoptera: Papers celebrating the $80^{\text {th }}$ birthday of Roy A. Crowson, J. Pakaluk y S. A. Slipinski. (eds.). Muzeum i Instytut Zoologii PAN, Warszawa. p. 7791006.

Leksono, A., N. Nakagoshi e Y. Isagi. 2005. The effects of forest disturbances on flying insect assemblages in Trawas, east Java. Tropics 14:335-343.

Lobo, J. M. y F. M. Piera. 2002. Searching for a predictive model for species richness of Iberian dung beetle based on spatial and environmental variables. Conservation Biology 16:158173.

Luna, I., A. Velázquez y E. Velázquez. 2001. México. In Bosques nublados del Neotrópico, M. Kappelle y A. Brown. (eds.). Instituto Nacional de Biodiversidad, San José [Costa Rica]. p. $183-229$.

Luna, I., J. J. Morrone y D. Espinosa. 2004. Biodiversidad de la sierra Madre Oriental. Comisión Nacional para el Conocimiento y Uso de la Biodiversidad / Universidad Nacional Autónoma de México, México, D.F. 527 p.

Luna, I., S. Ocegueda y O. Alcántara. 1994. Florística y notas biogeográficas del bosque mesófilo de montaña del municipio de Tlanchinol, Hidalgo, México. Anales del Instituto de Biología, Universidad Nacional Autónoma de México, Serie Botánica 65:31-62.

Magurran, A. E. 1988. Diversidad ecológica y su medición. Vedra, Barcelona. 200 p.

Manly, B. F. J. 1998. Multivariate statistical methods, segunda edición. Conservation Biology Series 9. Champan \& Hall, London. p. 233.

Márquez, J. 1994. Coleopterofauna asociada a detritos de Atta mexicana (F. Smith) (Hymenoptera: Formicidae) en dos localidades del norte de Morelos, México. Tesis, Facultad de Ciencias, Universidad Nacional Autónoma de México. México, D. F. 134 p.

Márquez, J. 1998. Staphylinidae (Insecta: Coleoptera) necrófilos del municipio de Tlayacapan, Morelos. Tesis maestría, Facultad de Ciencias, Universidad Nacional Autónoma de México, México, D. F. 166 p.

Márquez, J. 2003. Systematic revision of the genera Homalolinus and Ehomalolinus (Coleoptera, Staphylinidae, Xantholinini). Zoologica Scripta 32: 491-523.

Márquez, J. 2004. Colección de coleópteros del Centro de Investigaciones Biológicas, Universidad Autónoma del Estado de Hidalgo, Pachuca. 35 p.

Márquez, J. 2005. Técnicas de colecta y preservación de insectos. Boletín de la Sociedad Entomológica Aragonesa 37:385-408.

Márquez, J. 2006. Primeros registros estatales y datos de distribución geográfica de especies mexicanas de Staphylinidae (Coleoptera). Boletín de la Sociedad Entomológica Aragonesa 38:181-198.

Martínez, A. P. 2006. Relación entre diversidad taxonómica y funcional de meso y macrofauna: su influencia en la tasa de descomposición de la hojarasca en un bosque templado sujeto a manejo forestal. Tesis, Maestría en Recursos Bióticos, Instituto de Ciencias Básicas e Ingeniería, Universidad 
Autónoma del Estado de Hidalgo, Pachuca. 106 p.

May, R. M. 1988. How many species are there on earth? Science (Washington, D.C.) 241:1441-1449.

Mayr, E. 1992. A local flora and the biological species concept. American Journal of Botany 79:222-238.

McWilliam, H. A. y R. G. Death. 1998. Arboreal arthropod communities of remnant podocarp-hardwood rainforest in North Island, New Zealand. New Zealand Journal of Zoology 25:157-169.

Moreno, C. E. 2001. Métodos para medir la biodiversidad, vol. 1, M\&T-Manuales y Tesis, SEA, Zaragoza. 84 p.

Moreno, C. E. y G. Halffter. 2000. Assessing the compleness of bat biodiversity inventories using species accumulation curves. Journal of Applied Ecology 37:149-158.

Morón, M. A. 2003. Atlas de los escarabajos de México. Coleoptera Lamellicornia, vol. II. Familias Scarabaeidae, Trogidae, Passalidae y Lucanidae. Argania, Barcelona. 227 p.

Morón, M. A. y R. A. Terrón. 1984. Distribución altitudinal y estacional de los insectos necrófilos en la sierra Norte de Hidalgo, México. Acta Zoológica Mexicana (nueva serie) 3:1-47.

Morón, M. A. y R. A. Terrón. 1986. Coleópteros xilofilos asociados con Liquidambar styraciflua (L.) en la sierra de Hidalgo, México. Folia Entomológica Mexicana 67:25-35.

Morón, M. A., B. C. Ratcliffe y A. C. Deloya. 1997. Atlas de los escarabajos de México. Coleoptera Lamellicornia, I. Familia Melolonthidae. Sociedad Mexicana de Entomología, Veracruz, $280 \mathrm{p}$.

Morón-Ríos, A. y M. A. Morón. 2001. La fauna de Coleoptera Melolonthidae de la Reserva de la Biosfera "El Triunfo", Chiapas, México. Acta Zoológica Mexicana (nueva serie) $84: 1-25$.

Navarrete-Heredia J. L. y H. E. Fierros-López. 2001. Coleoptera de México: situación actual y perspectivas de estudio. In Tópicos selectos sobre Coleoptera de México, J. L. NavarreteHeredia, H. E. Fierros-López y A. Burgos-Solorio (eds.), Universidad de Guadalajara, Jalisco /Universidad Autónoma del Estado de Morelos, Cuernavaca. p. 1-21.

Navarrete-Heredia, J. L. y S. Zaragoza-Caballero. 2006. Diversidad de los Staphylinoidea de México: Análisis de grupos selectos (Hydraenidae, Agyrtidae, Silphidae y Staphylinidae). Dugesiana 13:53-65.

Navarrete-Heredia, J. L., A. F. Newton, M. K. Thayer, J. S. Ashe y D. S. Chandler. 2002. Guía ilustrada para los géneros de Staphylinidae (Coleoptera) de México. Illustrated guide to the genera of Staphylinidae (Coleoptera) of Mexico. Universidad de Guadalajara / Comisión Nacional para el Conocimiento y Uso de la Biodiversidad, Guadalajara, Jalisco. 401 p.

Odegaard, F. 2000. How many species of arthropods? Erwin's estimate revised. Biological Journal of the Linnean Society 71:583-597.

Peck, S. B. y A. E. Davies. E. 1980. Collecting small beetles with large-area "window" traps. The Coleopterists Bulletin
34:237-239.

Pérez, A. 1996. Coleopterofauna procedente del follaje de una selva baja caducifolia en la región de Chamela, Jalisco. Tesis, Facultad de Ciencias, Universidad Nacional Autónoma de México, México, D. F. 72 p.

Reyes-Novelo, E. y M. A. Morón. 2005. Fauna de Coleoptera Melolonthidae y Passalidae de Tzucacab y Conkal, Yucatán, México. Acta Zoológica Mexicana (nueva serie) 21:15-49.

Reyes-Novelo, E., H. Delfín-González y M. A. Morón. 2007. Copro-necrophagous beetle (Coleoptera: Scarabaeidae) diversity in an agroecosystem in Yucatan, Mexico. Revista de Biología Tropical 55:83-99.

Sánchez-Cordero, V., A. Townsend y P. Escalante-Pliego. 2001. El modelado de la distribución de especies y la conservación de la diversidad biológica. In Enfoques contemporáneos para el estudio de la biodiversidad, H. M. Hernández, A. N. García Aldrete, F. Álvarez y M. Ulloa. (comps.). Instituto de Biología, UNAM, México, D. F. p. 359-379.

SCT (Secretaría de Comunicaciones y Transportes). 1995. México. Atlas de Comunicaciones y Transportes. Escala 1: 500 000. Planeta, México, D.F.

SEDESOL (Secretaría de Desarrollo Social). 2002. Municipios de Hidalgo. Tlanchinol. Secretaría de Desarrollo Social, Pachuca, Hidalgo.

Soberón, J. y J. Llorente. 1993. The use of species accumulation functions for the prediction of species richness. Conservation Biology 7:480-488

Sokal, R. R. y P. H. A. Sneath. 1963. Principles of numerical taxonomy. Freeman, San Francisco, California.

Solís, A. 2002. Escarabajos de Costa Rica: las familias y subfamilias más comunes, segunda edición. Instituto Nacional de Biodiversidad, San José [Costa Rica]. 132 p.

Spellerberg, I. F. 1991. Monitoring ecological change. Cambridge University Press, 334 p.

Statsoft, Inc. 1998. Statistica for Windows (programa para computadora). Statsoft, Tulsa, Oklahoma.

Tóthmérész, B. 1995. Comparison of different methods for diversity ordering. Journal of Vegetation Science 6:283290.

Tóthmérész, B. 1998. On the characterization of scale-dependent diversity. Abstracta Botanica 22:149-156.

Wheeler, Q. D. 2004. Taxonomic triage and the poverty of phylogeny. Philosophical Transactions of the Royal Society of London 359:571-583.

Wheeler, Q. D., P. H. Raven, y E. O. Wilson. 2004. Taxonomy: impediment or expedient? Science (Washington, D.C.) 303:285.

White, R. E. 1983. A field guide to the beetles of North America. Houghton Mifflin, New York. 368 p.

Wolda, H. 1988. Insect seasonality: why? Annual Review of Ecology and Systematics 19:1-18.

Zar, J. H. 1996. Biostatistical analysis, tercera edición. Prentice Hall, Upper Saddle River, New Jersey. 867 p. 